\title{
Optimal Design of Reliable Integrated Chemical Production Sites
}

\author{
Sebastian Terrazas-Moreno, Ignacio E. Grossmann* \\ Chemical Engineering Department \\ Carnegie Mellon University \\ John M. Wassick, Scott J. Bury \\ The Dow Chemical Company \\ Rev. July 2010
}

* Author to whom correspondence should be addressed. Email: grossmann@cmu.edu, Tel: 412-268-3642, Fax: 412-268-7139. 


\begin{abstract}
Since plants that form the network are subject to fluctuations in product demand or random mechanical failures, design decisions such as adding redundant units and increasing storage between units can increase the flexibility and reliability of an integrated site. In this paper, we develop a bi-criterion optimization model that captures the trade-off between capital investment and process robustness in the design of an integrated site. Design decisions considered are increases in process capacity, introduction of parallel units, and addition of intermediate storage. The Mixed-integer Linear Programming (MILP) formulation proposed in this paper includes the representation of the material levels in the intermediate storage by means of a probabilistic model that captures the effects of the discrete, uncertain events. We also integrate a superstructure optimization with stochastic modeling techniques such as continuous time Markov chains. The application of the proposed model is illustrated with two example problems.
\end{abstract}




\section{Introduction}

An integrated site consists of a network of plants or large scale processes (Feord, 2002; Kimm, 2008; Wassick, 2009). The overall objective of the integrated site is to transform materials supplied from outside of the network into a set of products for which there is an external demand. The network structure is determined so that intermediate products can be internally produced by some plants and consumed by others.

Most large-scale chemical integrated sites, such as Dow Texas Operations, have gradually evolved from smaller sites. The opportunity to design and build completely new integrated chemical sites has appeared fairly recently. The size and scope of some of these projects has seldom, if ever, been done before. The risk of integration failures (mismatch on capacity, blocking or starving other units, etc.) has a much larger downside than with traditional, independent production plants. Of particular concern is the fact that uncertain events affect the performance of an integrated site. Some of these are discrete, such as process failures, while others are continuous, such as variations in external supply, demand, or fluctuations in plant throughput. Under such uncertain conditions, the design of integrated sites presents challenges for smooth integration and balancing of process availability and capacity. Some design decisions can increase the robustness of the integrated site subject to continuous and discrete uncertainties.

The objective of this work is to develop a systematic method to determine the optimal trade-off between capital investment and process robustness in the design of an integrated site subject to discrete and continuous uncertainties. The main design decisions considered are changes in process capacity, introduction of parallel units, and addition of intermediate storage. We use a metric known as expected stochastic flexibility $\mathrm{E}(\mathrm{SF})$ to quantify the robustness of the integrated site. $\mathrm{E}(\mathrm{SF})$ is a probabilistic measure of the system's ability to tolerate continuous and discrete uncertainties (Straub \& Grossmann, 1990).

Other authors have studied the problem of addressing reliability or availability of a process at the design stage. Pistikopoulos et al. (1996) addressed the simultaneous design and maintenance optimization problem. They introduced a flexibility-reliability index (FRI) that has the same definition as the expected stochastic flexibility of Straub \& Grossmann (1990). Pistikopoulos et al. (2001) simultaneously solved the production 
planning and maintenance planning problem for multipurpose batch process plants. They define system effectiveness as the criterion that balances increased revenues from process availability improvements and increased maintenance. In a later work, Goel et al. (2003) extended the system effectiveness approach to incorporate the initial availability of each process in the system as a degree of freedom. Sherali et al. (2008) studied the optimal allocation of risk-reduction resources using an event tree representation. Some resources are used to prevent events in the tree from happening, while others are used to minimize the loss once the events occur.

Straub \& Grossmann (1993) proposed a mathematical formulation for maximizing stochastic flexibility (SF) at the design level. In this approach, the feasible region is determined by the choice of the design variables. The stochastic flexibility is approximated using a set of quadrature points that are placed adaptively inside the feasible region. This approach requires the solution of a large nonlinear program (NLP). Bansal et al. $(2000,2002)$ proposed a parametric programming approach to quantify stochastic flexibility. The authors derive a set of parametric solutions dependent on design and uncertain parameters, and a corresponding set of regions where those solutions are optimal.

Davies and Swartz (2008) studied the effect of intermediate storage on the performance of a continuous process under random failures. Their approach relies on generating failure scenarios and optimizing buffer levels over these scenarios using an economic objective function. Cheng et al. (2003) addressed the problem of design and planning under uncertainty. Their work is similar to the one presented in this paper in that both involve multi-objective optimization under uncertainty. They modeled discrete uncertainties using a Markov decision model where the states of the system depend on external uncertain conditions (available processing technologies) and internal control decisions (number of processing units and type of catalyst). A two-stage stochastic programming problem is solved at each discrete state, where the first stage decisions are given by the process design, and the second stage decisions are related with production planning. These authors also included the possibility of modifying the design of the process in different time periods as more information becomes available. Our work addresses some points not considered by Cheng et al. (2003). We consider inventory as a 
way of hedging against uncertainty, and we allow the infeasible operation through the use of the stochastic flexibility index. Also, the source of uncertainty in our work is both external (demand and supply) and internal (random unit failures).

The work presented in this paper makes three contributions to the related fields. First, it proposes a bi-criterion Mixed-integer Linear Programming (MILP) formulation for the maximization of expected stochastic flexibility $\mathrm{E}(\mathrm{SF})$ and minimization of investment cost. For the evaluation of the expected stochastic flexibility, the basic idea relies on considering fixed quadrature points and using auxiliary 0-1 variables to determine feasibility/infeasibility of operation. Second, it integrates the effect of intermediate storage to the design framework for evaluating and optimizing expected stochastic flexibility $\mathrm{E}(\mathrm{SF})$, a nontrivial task given that no explicit timing considerations are included in existing formulations for optimizing either the E(SF) or the FRI. The basic idea here relies on the use of Markov chains and basic random process theory to capture the discrete-event nature of the problem. Third, in contrast to considering a fixed network structure as has been done in the past, our work also considers the integration of superstructure optimization with the Markov chain model of discrete events in the determination of optimal $\mathrm{E}(\mathrm{SF})$. We illustrate our approach with a simple example from the literature and with an industrial case study. As a result of applying our methodology to these examples, trade-off curves of optimal expected stochastic flexibility vs. capital investment are obtained.

\section{Background}

Stochastic flexibility

The general mathematical model of a process can be defined as follows:

$h(x, d, \theta, y)=0$

$g(x, d, \theta, y) \leq 0$

where

$x:$ continuous process variables

$d:$ design variables

$\theta:$ uncertain parameters

$y:$ binary parameter used to include the effect of failures in the processing units. 
In this work, we only deal with linear equations and inequalities. An example of such a case is shown in Figure 1 where $\mathcal{F}$ corresponds to the feasible region. The contours of the joint probability distribution for uncertain parameters $\theta_{1}$ and $\theta_{2}$ are the ellipses in the figure. The stochastic flexibility (SF) is the cumulative probability of the joint distribution that lies within the feasible region (Straub \& Grossmann, 1990).

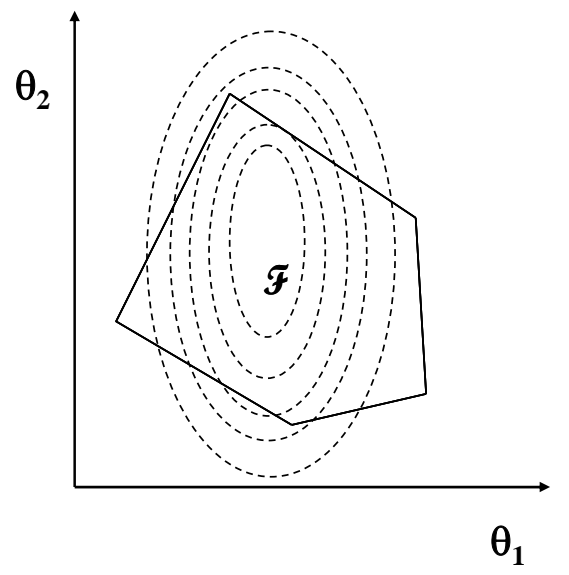

Fig. 1 Stochastic flexibility of a feasible region $F$ defined by a set of linear constraints

The size of $\mathscr{F}$ is a function of the design variables. In the case of an integrated site; parallel units, extra capacity additions, and intermediate storage, have the potential to enlarge the size of $\mathscr{F}$ (i.e. operation is feasible for a larger range of uncertain parameter values).

\section{Stochastic Processes}

A stochastic process is a collection of random variables that are all defined on the same probability space and indexed by a real parameter (Heyman and Sobel, 1982). We denote a stochastic process by $\{X(t) ; t \in T\}$, where $T$ is a set of numbers that indexes the random variable $X(t)$. For this work, $t$ refers to time and $T$ to the range of times being considered. In this context, $X(t)$ is the value of the process at time $t$. When $T$ is finite or countable infinite, it is said to be discrete; otherwise it is called continuous. The state space of the process $S$ is the set of possible values for $X(t)$. We call the values $s \in S$ states. When $S$ contains a finite or countable infinite number of states, it is said to be discrete, otherwise, it is called continuous. For any state $s \in S$ and any time $t \in T$, if $X(t)=s$, we say that the process is in state $s$ at time $t$. The terms stage and epoch are sometimes substituted for time, especially in discrete time processes. 


\section{Continuous time Markov chain}

The stochastic process $\{X(t) ; t \geq 0\}$ is a continuous-time Markov chain with state space $S \subset$ Integers if each $X(t)$ assumes values only in $S$ and

$P\left\{X\left(t_{n+1}\right)=j \mid X\left(t_{0}\right)=i_{0}, \ldots, X\left(t_{n}\right)=i_{n}\right\}=P\left\{X\left(t_{n+1}\right)=j \mid X\left(t_{n}\right)=i_{n}\right\}$

where $P\{\bullet\}$ represents the probability of the event inside the brackets. The conditional probability notation $P\{A \mid B\}$ means the probability of $A$ given $B$. This is the memory less property; that is, the future states of a system are independent of all past states except for the immediately preceding one (Billinton and Allan, 1992).

Availability problems in process networks subject to random failures can be modeled as continuous time Markov chains. The state space of these systems is made up of all the different combinations of failures in the network. Each one of these combinations is called a discrete state. For example, a two processing unit network is subject to random failures that can completely shut down each of the two units. Failure $1\left(F_{1}\right)$ affects the first unit and failure $2\left(F_{2}\right)$ the second. The state space of the system is $S=\left\{(\right.$ no failure $),\left(F_{1}\right),\left(F_{2}\right),\left(F_{1}\right.$ and $\left.\left.F_{2}\right)\right\}$; each of the elements in $S$ is a state. The system transitions between states when a failure occurs or when a failure is repaired. In continuous Markov chains, each transition is described by a rate. The probability that the system transitions from the (no failure) state to the $\left(F_{2}\right)$ state depends on the probability of failure of the second unit. When the failure probability is described by an exponential distribution, then the failure rate of that distribution corresponds to the transition rate from the (no failure) state to the $\left(F_{2}\right)$ state. Figure 2 shows a useful way of representing the continuous time Markov chain by means of a state space diagram and its corresponding transitions. If the states of a system and the rates of transition between them are specified (failure and repair rates), we can use straightforward and well known "frequency" and "duration" techniques to obtain important information about the system (Billinton and Allan, 1992). Namely, for state $s$, we can calculate the long term probability $\left(\mathrm{prob}^{s}\right)$ of finding the state, mean residence time $\left(m r t^{s}\right)$, frequency of encounters $\left(f r^{s}\right)$, and the cycle time for the reappearance of the state $\left(t c^{s}\right)$. We illustrate these techniques for the two-unit system in Figure 2. 


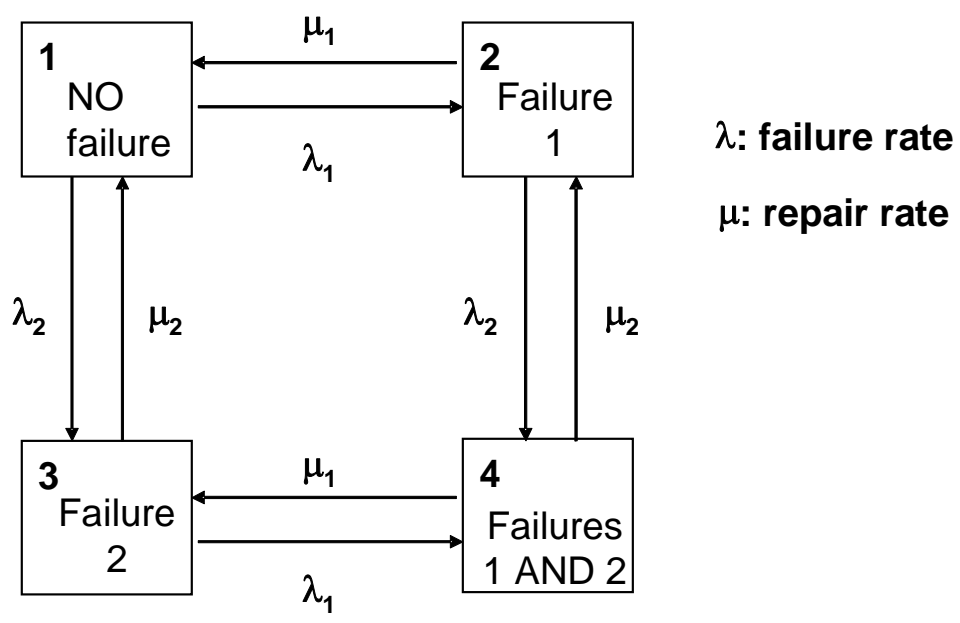

Fig. 2 Space state diagram for a two component system

Suppose the two units in the system are subject to one type of random failure that shuts them down completely. The time to failure $T T F_{m}$ and time to repair $T T R_{m}$ for each unit $m$ are probabilistic quantities that follow an exponential distribution. As part of the reliability data of the system we are given the mean time to failure $M T T F_{m}$ and mean time to repair $M_{T T R_{m}}$. The failure rate $\lambda_{m}$ and repair rate $\mu_{m}$ that characterize the exponential distributions are the reciprocals of the $M_{T T F}$ and $M T T R_{m}$ respectively. The repair rate represents the total number of repairs divided by the total down time. The equations that allow us to calculate $p r o b^{s}, m r t^{s}, f r^{s}$, and $t c^{s}$ from the knowledge of the possible system states (Figure 2) and the transition rates between them $\left(\lambda_{m}\right.$ and $\left.\mu_{m}\right)$, as presented by Billinton and Allan (1992), are as follows.

The probability of finding any unit $m$ in a failed state is $\frac{M T T R_{m}}{M T T R_{m}+M T T F_{m}}=\frac{\lambda_{m}}{\lambda_{m}+\mu_{m}}$, and the probability of finding it active is $\frac{M T T F_{m}}{M T T R_{m}+M T T F_{m}}=\frac{\mu_{m}}{\lambda_{m}+\mu_{m}}$. The states in Figure 2 involve combinations of failed and active units. The probability of finding each of the states in the figure can be calculated by independent combinations, as shown below: 


$$
\begin{aligned}
& \operatorname{prob}^{1}=\frac{\mu_{1} \mu_{2}}{\left(\lambda_{1}+\mu_{1}\right)\left(\lambda_{2}+\mu_{2}\right)} \\
& \operatorname{prob}^{2}=\frac{\lambda_{1} \mu_{2}}{\left(\lambda_{1}+\mu_{1}\right)\left(\lambda_{2}+\mu_{2}\right)} \\
& \operatorname{prob}^{3}=\frac{\mu_{1} \lambda_{2}}{\left(\lambda_{1}+\mu_{1}\right)\left(\lambda_{2}+\mu_{2}\right)} \\
& \operatorname{prob}^{4}=\frac{\lambda_{1} \lambda_{2}}{\left(\lambda_{1}+\mu_{1}\right)\left(\lambda_{2}+\mu_{2}\right)}
\end{aligned}
$$

The frequency of encountering each state $s, f r^{s}$, is calculated as the probability of finding $s, \operatorname{prob}^{s}$, times the rate of departure from $s$. The rate of departure is the summation of all the rates that leave the state. For instance, the rate of departure from state 1 in Figure 2 is $\lambda_{1}+\lambda_{2}$. Thus,

$f r^{1}=\frac{\mu_{1} \mu_{2}}{\left(\lambda_{1}+\mu_{1}\right)\left(\lambda_{2}+\mu_{2}\right)}\left(\lambda_{1}+\lambda_{2}\right)$

$f r^{2}=\frac{\lambda_{1} \mu_{2}}{\left(\lambda_{1}+\mu_{1}\right)\left(\lambda_{2}+\mu_{2}\right)}\left(\mu_{1}+\lambda_{2}\right)$

$f r^{3}=\frac{\mu_{1} \lambda_{2}}{\left(\lambda_{1}+\mu_{1}\right)\left(\lambda_{2}+\mu_{2}\right)}\left(\lambda_{1}+\mu_{2}\right)$

$f r^{4}=\frac{\lambda_{1} \lambda_{2}}{\left(\lambda_{1}+\mu_{1}\right)\left(\lambda_{2}+\mu_{2}\right)}\left(\mu_{1}+\mu_{2}\right)$

The cycle time between individual states $\left(t c^{s}\right)$ is the reciprocal of the frequency of encounters:

$t c^{s}=\frac{1}{f r^{s}}$

Finally, the mean duration or residence time in state $s\left(m r t^{s}\right)$ is calculated as the probability of finding $s$ divided by the frequency of encounters:

$$
m r t^{s}=\frac{p r o b^{s}}{f r^{s}}
$$

\section{Problem Definition}


The problem that we address in this paper can be stated as follows. We are given a set of finished products with a demand that is either deterministic, or given by a specified probability distribution. The raw materials and the intermediate products involved in the integrated production site are known. The supply of raw material can also be deterministic, or given by a specified probability distribution. There are a predetermined number of steps involved in the transformation of raw materials to intermediate and finished products. Each of these steps is carried out by a different plant that can have multiple production units. Plants are connected through the flow of intermediate products between them. The network formed by the plants and their connections represents an integrated site as shown in the example in Figure 3.

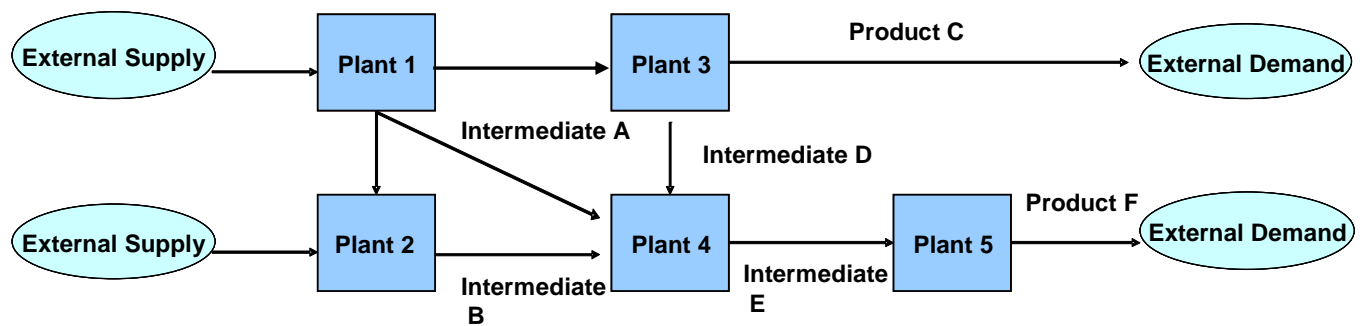

Fig. 3 Integrated Site for the production of Products C and F.

The plants in the integrated site are subject to random failures that result in corrective maintenance. As a result, plants experience some amount of down time during which production is decreased or stopped all together. Since plants are interconnected in the integrated site, a failure event will propagate downstream or upstream, forcing some other plants to decrease or stop their production. In Figure 4 the dotted lines correspond to the flows that are affected by the failure of Plant 4 . If these events are not considered while designing the integrated site, there is a risk that product demand will not be consistently met.

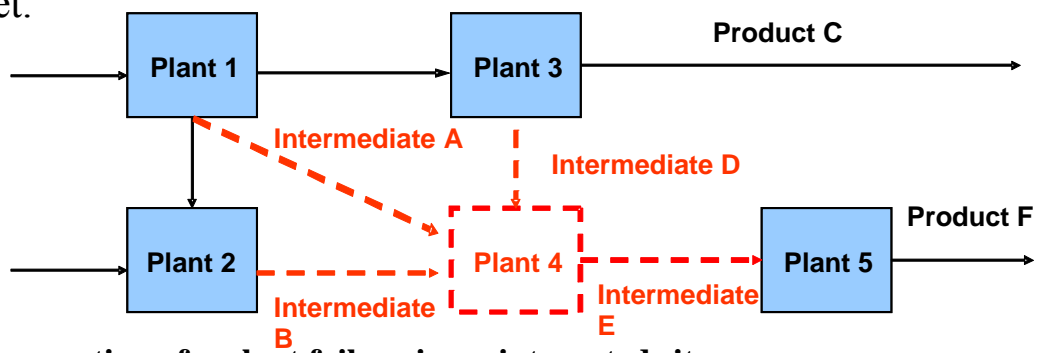

Fig. 4 Propagation of a plant failure in an integrated site 
We consider three types of design variables that have an impact on the flexibility and reliability of an integrated site. These variables are the sizing of intermediate storage, the potential addition of parallel production units to each plant, and the increase of plant capacities. Figures 5 and 6 illustrate addition of parallel units and intermediate storage.

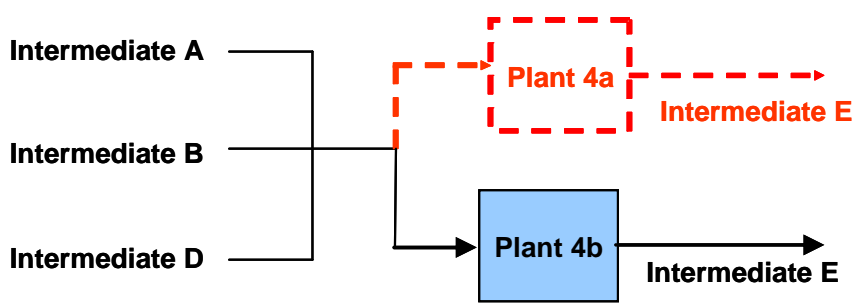

Fig. 5 Process redundancies

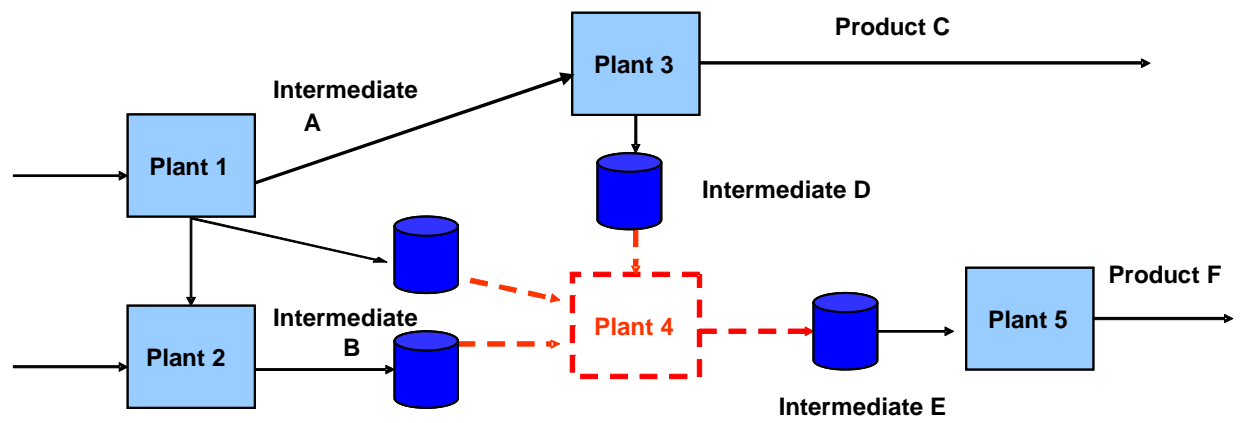

Fig. 6 Intermediate storage between plants in the network

A well designed integrated site maximizes the probability of consistently meeting the production requirements for a fixed available capital investment.

The problem can be stated more precisely as follows.

We are given:

- The superstructure of an integrated site with all allowable parallel production units in each plant and intermediate storage tanks.

- A set of materials that the plants consume and produce.

- Mass balance coefficients for all units in the superstructure.

- Base processing capacity and a range for extra capacity increments for each unit.

- Supply of raw materials and demand for finished products (constant or described by a probability distribution). 
- Number of failures, associated production rate reductions, and the mean time to failure MTTF and mean time to repair MTTR that completely specify the exponential distributions of failure and repair times.

- A set of frequency and duration equations that allows us to calculate the cycle time $t c$, mean residence time $m r t$, and frequency of encounters $f r$, for all the possible states in the system, based only on the knowledge of the MTTR and MTTF of the units in the superstructure (Billinton and Allan, 1992). These equations were explained in the background section of this paper.

- A continuous range of tank sizes for intermediate storage.

- A cost function that relates design decisions with capital investment.

The problem is to determine:

- For each plant, the number of production units.

- For each plant, the capacity increment for each unit above the base capacity.

- Sizes of intermediate storage between plants.

Subject to:

- Mass balances.

- Process specifications.

- Bounds on production capacity and tank sizes.

The objective is to determine the set of Pareto-optimal solutions that:

- Maximize the expected stochastic flexibility.

- Minimize the capital investment.

The following assumptions and simplifications are made:

1. Unplanned down time is the result of random and independent failure events.

2. TTF and TTR have exponential distributions. In the case that TTR follows a normal distribution the Markov chain approach is no longer rigorously valid. However, we claim that the Markov chain model is a useful approximation for analyzing the long term behavior of the Integrated Site. We quote Billinton \& Allan (1992) on this point: "It has been stressed [...] that, although the assumption of exponential distributions may have been made, the results and equations are equally applicable to all 
distributions if only the limiting state or long term average values are being evaluated for systems containing statistically independent components."

3. Preventive maintenance is not included.

4. The design cost function is linear.

5. Each plant can be modeled in terms of one output product. The production of all other products is proportional to the main output. Mass balance coefficients can be used to specify that the main product can only use a fraction of the total plant capacity.

Maximizing the expected stochastic flexibility and minimizing the capital investment yields a bi-criterion optimization problem. The results of this bi-criterion optimization in the form of a Pareto-optimal curve can be obtained by maximizing the $\mathrm{E}(\mathrm{SF})$ for various fixed values of capital investment (i.e. $\varepsilon$-constrained method (Ehrgott, 2005)).

\section{Mathematical model}

\section{Maximization of expected stochastic flexibility}

In the background section, we reviewed the concept of stochastic flexibility (SF). In this section, we introduce expected stochastic flexibility $\mathrm{E}(\mathrm{SF})$ as presented by Straub and Grossmann (1990). We then describe our new approach to maximizing E(SF) in process systems. For the sake of simplicity in the notation, we use only two uncertain parameters $\left(\theta_{1}, \theta_{2}\right)$ described by a joint probability distribution $j\left(\theta_{1}, \theta_{2}\right)$. The two uncertain parameters will be discretized using $k_{1}\left(k_{1}=1,2, \ldots\left|K_{1}\right|\right)$ and $k_{2}\left(k_{2}=1,2, \ldots\left|K_{2}\right|\right)$ quadrature points. Any point in the $\left(\theta_{1}, \theta_{2}\right)$ space is described by a pair $\left(k_{1}, k_{2}\right)$, where $k_{1}$ and $k_{2}$ denote the indices of the quadrature points for $\theta_{1}$ and $\theta_{2}$ respectively.

Discrete uncertainties related to possible failures of the production units are captured through the discrete states $s(s=1,2, \ldots,|S|)$. Each discrete state is described by a set of failures in the processing units in the system. Mathematically this is represented by the binary parameter vector $y^{s}$ that has as many elements as the total number of possible 
failures in all the units of the system. A zero value in a certain position of the $y^{s}$ vector denotes that a failure is present or occurring when the system is in discrete state $s$.

Equations (1) and (2) describe a general process model. These equations can be written for each point $\left(k_{1}, k_{2}\right)$ and each discrete state $s$ :

$h_{k 1 k 2}^{s}\left(x_{k 1 k 2}^{s}, d, \theta_{1_{k 1}}, \theta_{2 k 2}, y^{s}\right)=0$

$g_{k 1 k 2}^{s}\left(x_{k 1 k 2}^{s}, d, \theta_{1_{k 1}}, \theta_{2_{k 2}}, y^{s}\right) \leq 0$

We introduce a non-negative slack variable $u$, and a binary variable $w$ associated with each discrete point $\left(k_{1}, k_{2}\right)$ in each state $s$. If the point $\left(k_{1}, k_{2}\right)$ lies inside the feasible region corresponding to state $s$, the binary variable $w_{k 1, k 2}^{s}$ takes a value of one and the slack is zero as given by equations (10) - (14). Infeasibility leads to a nonzero slack, which in turn removes inequality (12). The parameter $M$ in equation (12) corresponds to a valid upper bound for the left hand side of equation (11).

For each

$k_{1}=1,2, \ldots\left|K_{1}\right| ; k_{2}=1,2, \ldots\left|K_{2}\right| ; s=1,2, \ldots,|S|$

we have:

$$
\begin{aligned}
& h_{k 1 k 2}^{s}\left(x_{k 1 k 2}^{s}, d, \theta_{1_{k 1}}, \theta_{2_{k 2}} y^{s}\right)=0 \\
& g_{k 1 k 2}^{s}\left(x_{k 1 k 2}^{s}, d, \theta_{1_{k 1}}, \theta_{2_{k 2}} y^{s}\right) \leq u_{k 1 k 2}^{s} \\
& u_{k 1 k 2}^{s} \leq M\left(1-w_{k 1 k 2}^{s}\right) \\
& u_{k 1 k 2}^{s} \geq 0 \\
& w_{k 1 k 2}^{s} \in\{0,1\}
\end{aligned}
$$

Assuming a given quadrature formula for approximating the integral in the stochastic flexibility (e.g. Gaussian quadrature), we calculate the probability $\gamma_{k 1 k 2}$ associated with each point $\left(k_{1}, k_{2}\right)$ as follows:

$$
\gamma_{k 1 k 2}=\omega_{k 1} \omega_{k 2} \frac{\left(\theta_{1}^{U}-\theta_{1}^{L}\right)}{2} \frac{\left(\theta_{2}^{U}-\theta_{2}^{L}\right)}{2} \times j\left(\theta_{1_{k 1}}, \theta_{2_{k 2}}\right)
$$

where $\omega_{i}$ is the weight of the $i-t$ th quadrature point. $\theta_{i}^{L}, \theta_{i}^{U}$ are lower and upper bounds of the uncertain parameters. Note that these bounds are independent of the feasible 
region. For instance, normally distributed parameters can be bounded by the average value minus and plus four standard deviations. $j\left(\theta_{1_{k 1}}, \theta_{2_{k 2}}\right)$ is the value of the joint probability distribution at the point $\left(k_{1}, k_{2}\right)$.

For a given state $s$, the stochastic flexibility can be computed as:

$S F^{s}=\sum_{k 1=1}^{K 1} \sum_{k 2=1}^{K 2} \gamma_{k 1 k 2}^{s} w_{k 1 k 2}^{s}$

Figure 7 illustrates our approach for calculating the stochastic flexibility.

The objective of maximizing the expected stochastic flexibility $\mathrm{E}(\mathrm{SF})$ can now be defined as follows,

$$
\max E(S F)=\sum_{s=1}^{S} \operatorname{prob}^{s} \sum_{k 1=1}^{K 1} \sum_{k 2=1}^{K 2} \gamma_{k 1 k 2}^{s} w_{k 1 k 2}^{s}
$$

Here $\operatorname{prob}^{s}$ is the probability of finding the system in discrete state $s$ after a long time of operation. $\operatorname{prob}^{s}$ can be calculated from the reliability data of the integrated site; it is a parameter in the optimization formulation. Equation (17) is used as one objective function for the bi-criterion formulation developed in this work.

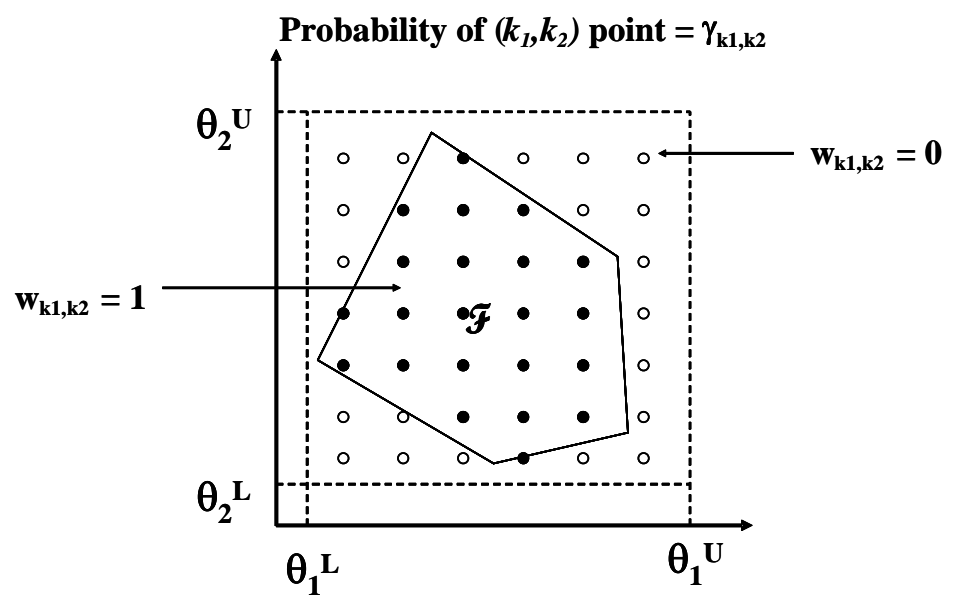

Fig. 7 Stochastic flexibility by discretization of uncertain parameters

The formulation described by equations (10) - (14) and (17) results in a problem that grows exponentially with the number of uncertain parameters and number of elements in the $y$ vector (i.e., the number of possible failures in the integrated site). Therefore, algorithmic techniques are required for the solution of large-scale problems with this 
formulation. The need to check feasibility for every state and every collocation point might be avoided by using bounding search procedures (Straub and Grossmann, 1990) and logic cuts (Hooker et al., 1994). The focus of this paper, however, is the development of our formulation and its integration with intermediate storage modeling and superstructure optimization. We will address the solution of large-scale problems in a future paper.

\section{Integrated site process model}

So far we have used a general process model. In this section, we describe the particular model we use for integrated sites. The continuous process variables $x$ in equations (1) and (2) are material flows. As seen in Figure 8, the variable ps corresponds to the process streams that feed the units; the variables $f$ and flow are the remaining flow variables ( flow are inputs/outputs for each plant, $f$ are internal flows). The design variables $d$ in (1) and (2) correspond to production unit extra capacity additions $p c$, the binary variable associated with installation of a parallel production unit $z$, the storage tank volume $v$ and the average inventory level inv. The yield coefficients, the base processing capacity of each unit, and upper and lower bounds for all variables are deterministic parameters. The uncertain parameters $\theta$ in (1) and (2) are the raw material supply $R M$ and demand of finished products $D F$. In the following section, we describe the process network model for the integrated site corresponding to equations (8) and (9).

Let $j=1,2, \ldots,|J|$ refer to a set of large-scale chemical processes or plants, and $n=1,2, \ldots,|N|$ refer to the products consumed and produced by all the plants. The superstructure of the integrated site considers $m=1,2, \ldots,\left|M_{j}\right|$ parallel production units for each plant $j$. Every plant $j$ has an intermediate storage tank for product $n$, described by its total volume $v_{j, n}$ and its average inventory level $i n v_{j, n}$. Both of these quantities will take the value of zero if no storage is required. Figure 8 shows the basic building block for every plant $j$ in the superstructure. We use the variable $f_{j}$ to represent material flows within the block corresponding to plant $j$ (e.g. the flow from the production units to the storage tank) and flow $_{j^{\prime}, j}$ to represent the flow that goes from 
plant $j^{\prime}$ to plant $j$. The variable $p s_{m}$ stands for processing stream fed to each production unit $m$.

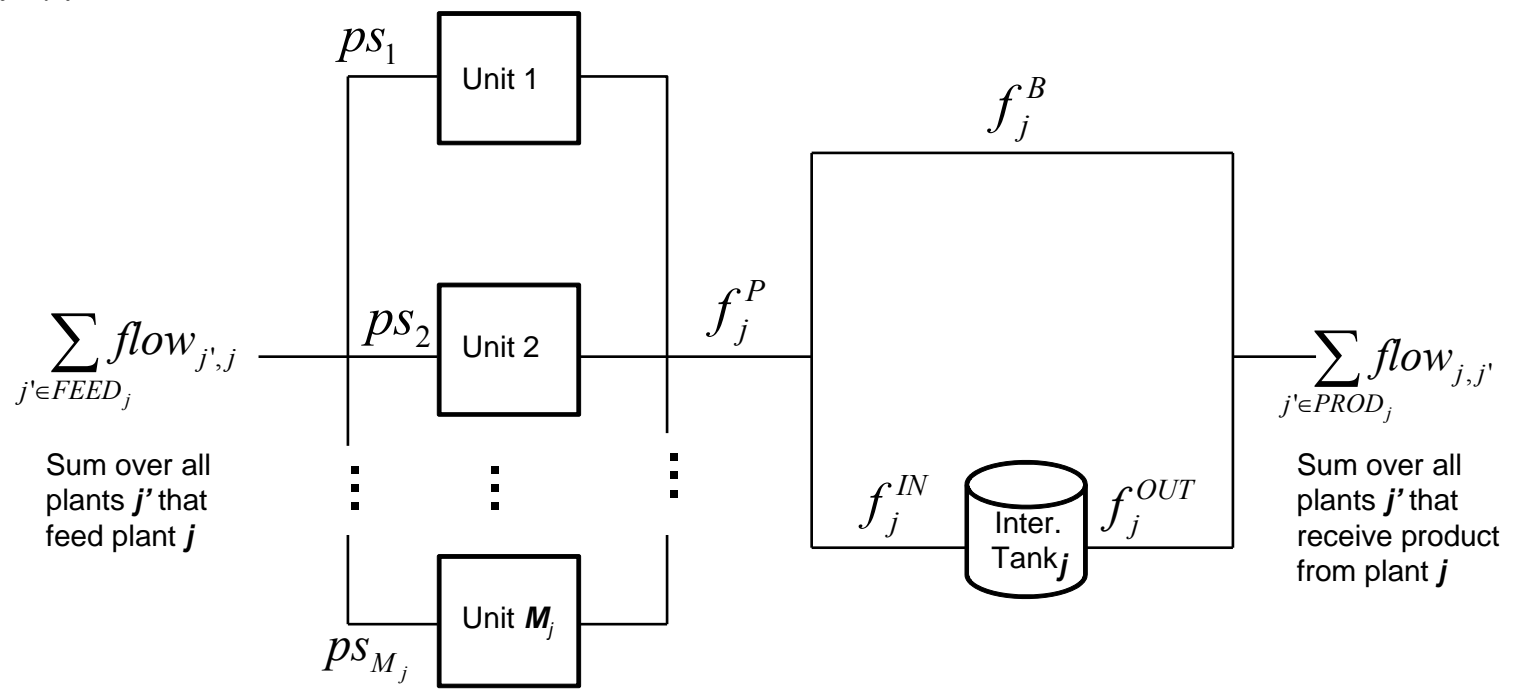

Fig. 8 Building block for plant $j$ in the integrated site

Finally, we have a set of $s=1,2, \ldots,|S|$ discrete system states, and sets $k_{1}=1,2, \ldots,\left|K_{1}\right|$ and $k_{2}=1,2, \ldots,\left|K_{2}\right|$ of quadrature points for uncertain parameters $\theta_{1}$ and $\theta_{2}$. The following equations describe the material balance for the building block shown in Figure 8.

$$
\begin{aligned}
& \sum_{j^{\prime} \in F E E D_{j}}\left[\sum_{n \in O U T P U T_{j^{\prime}} \cap I N P U T_{j}} \text { flow }_{j^{\prime}, j, n, s, k 1, k 2}\right]=\sum_{m \in M_{j}} p s_{m, s, k 1, k 2} \quad \forall j \in J, s \in S, k_{1} \in K_{1}, k_{2} \in K_{2} \\
& \frac{\sum_{j^{\prime} \in F E E D_{j}} \text { flow }_{j^{\prime}, j, n, s, k 1, k 2}}{\sum_{j^{\prime} \in F E E D_{j}} \text { flow }_{j^{\prime}, j, \bar{n}, s, k 1, k 2}}=\vartheta_{j, n}^{\bar{n}} \quad \forall j \in J, n \in N, \bar{n} \in \text { Nref }_{j}, s \in S, k_{1} \in K_{1}, k_{2} \in K_{2} \\
& \sum_{m \in M_{j}} \alpha_{m} p s_{m, s, k 1, k 2}=f_{j, n, s, k 1, k 2}^{P} \quad \forall j \in J, n \in \text { OUTPUT }_{j}, s \in S, k_{1} \in K_{1}, k_{2} \in K_{2} \\
& f_{j, n, s, k 1, k 2}^{P}=f_{j, n, s, k 1, k 2}^{I N}+f_{j, n, s, k 1, k 2}^{B} \quad \forall j \in J, n \in \text { OUTPUT }_{j}, s \in S, k_{1} \in K_{1}, k_{2} \in K
\end{aligned}
$$

$$
\text { flow }_{j^{\prime}, j, n, s, k 1, k 2}=0 \quad \forall j^{\prime} \in J, j \in J, n \notin O U T P U T_{j^{\prime}}, s \in S, k_{1} \in K, k_{2} \in K
$$


In equation (18) the set $F E E D_{j}$ contains all upstream plants that feed material to plant $j \quad$ INPUT $_{j}$ and $O U T P U T_{j}$ are subsets of $N$ corresponding to the materials consumed and produced by plant $j$. The set $M_{j}$ contains the parallel units $m$ postulated for plant $j$. The variable flow $_{j^{\prime}, j, n, s, k 1, k 2}$ represents the flow of product $n$ from plant $j^{\prime} \in F E E D_{j}$ to plant $j \in J$, during state $s$ and in quadrature point $\left(k_{1}, k_{2}\right)$. The variable $p s_{m, s, k 1, k 2}$ represents the flow supplied to the $m^{\text {th }}$ production unit in plant $j$ ( since $m \in M_{j}$ ) during state $s$ and in the quadrature point $\left(k_{1}, k_{2}\right)$. In equation (19), $\vartheta_{j, n}^{\bar{n}}$ is the mass balance coefficient for product $n$ in plant $j$ using product $\bar{n}$ as reference. For example, if plant 1 consumes two tons of raw material $\mathrm{B}$ for each ton of raw material $\mathrm{A}$, then $\vartheta_{1, A}^{A}=1$ and $\vartheta_{1, B}^{A}=2$. The parameter $\alpha_{m}$ in equation (20) is the yield coefficient for production unit $m$, while the variable $f_{j, n, s, k 1, k 2}^{P}$ represents the total flow of product $n \in$ OUTPUT $_{j}$ out of the $|M|$ parallel units in plant $j$. In equation (21), the flow into the storage tank is represented by $f_{j, n, s, k 1, k 2}^{I N}$. The flow that bypasses the storage tank is represented by $f_{j, n, s, k 1, k 2}^{B}$.

In equation (22), the set $P R O D_{j}$ contains all the plants that use the product of plant $j$. The variable $f_{j, n, s, k 1, k 2}^{O U T}$ represents the flow out of the storage tank. The net flow from plant $j$ to other plants $j^{\prime} \in P R O D_{j}$ in the network is $f l o w_{j, j^{\prime}, n, s, k 1, k 2}$.Equation (23) sets equal to zero the flows of the products $n$ that are not produced by plant $j^{\prime}$.

More constraints can be added for process specifications. The most common of them is the specification to consume no more than the maximum supply of raw materials and to produce at least all the finished product that is demanded. These constraints are written below.

$$
\begin{gathered}
\sum_{j \in P R O D_{\text {supplier }}} \text { flow }_{\text {supplier }, j, n, s, k 1, k 2} \leq R M_{n, k 1} \quad \forall n \in \mathrm{RAW} \subset N, s \in S, k_{1} \in K_{1}, k_{2} \in K_{2} \\
\sum_{j \in F E E D_{\text {consumer }}} \text { flow }_{j, \text { consumer }, n, s, k 1, k 2} \geq D F_{n, k 2}
\end{gathered}
$$


The set RAW is a subset of products corresponding to externally supplied raw materials. The set FINISHED contains the finished products sent to external consumers. The model considers one external supplier and one external consumer per product $n$. In practical problems there might be many suppliers and consumers, but we assume that their behavior can be summed into one large supplier and one large consumer. The sets $P R O D_{\text {supplier }}$ and $F E E D_{\text {consumer }}$ include the plants that use the externally supplied raw materials and that feed the external consumer, respectively. The supply of raw material $n \in \mathrm{RAW}$ to plant $j$ is labeled flow $w_{\text {supplier }, j, n}$ and the flow of finished product $n \in$ FINISHED from plant $j$ is labeled flow $_{j, \text { consumer }, n}$.

$R M_{n, k 1}$ is a parameter that indicates the external supply of $n$ in collocation point $k_{l}$; $D F_{n, k 2}$ is a parameter that indicates the amount of finished product $n$ demanded in collocation point $k_{2}$. The non-negative slack variable $u_{k 1, k 2}^{s}$ is added to equations (24) and (25), and the feasibility of the point $\left(k_{1}, k_{2}\right)$ in state $s$ is determined through the use of the binary variable $w_{k 1, k 2}^{s}$.

$\sum_{j \in P R O D_{\text {suppier }}}$ flow $_{\text {supplier }, j, n, s, k 1, k 2}-R M_{n, k 1} \leq u_{s, k 1, k 2} \quad \forall n \in \mathrm{RAW}, s \in S, k_{1} \in K_{1}, k_{2} \in K_{2}$

$D F_{n, k 2}-\sum_{j \in F E E D_{\text {consumer }}}$ flow $_{j, \text { consumer }, n, s, k 1, k 2} \leq u_{s, k 1, k 2} \forall n \in$ FINISHED, $s \in S, k_{1} \in K_{1}, k_{2} \in K_{2}$

$u_{k 1 k 2}^{s} \leq M\left(1-w_{k 1 k 2}^{s}\right) \quad \forall s \in S, k_{1} \in K_{1}, k_{2} \in K$

$u_{k 1, k 2}^{s} \geq 0 \quad \forall s \in S, k_{1} \in K_{1}, k_{2} \in K$

$w_{k 1 k 2}^{s} \in\{0,1\} \quad \forall s \in S, k_{1} \in K_{1}, k_{2} \in K$

\section{Representation of random failures}

Plant $j \in J$ in the integrated site can consist of multiple parallel production units $m \in M_{j}$. Let $M=\bigcup_{j \in J} M_{j}$ be the set of all units in the integrated site. Every unit $m \in M$ is subjected to random failures. Some of them are partial failures that decrease the production rate, while others are total failures that cause complete unit shutdown. Let $\ell=1,2, \ldots,\left|L_{m}\right|$ be the different types of random failures that arise in unit $m$. The set 
$L=\bigcup_{m \in M} L_{m}$ contains the possible failures of all the units in the integrated site. Each failure $\ell \in L$ is characterized by a production rate decrease (or rate cut) $r c_{\ell}$, an exponential distribution for time to failure $T T F_{\ell}$, and a normal or exponential distribution for time to repair $T T R_{\ell}$. Recall that in the Problem Definition section we presented the Markov chain model as a useful approximation of the average behavior of the system for long operating horizons, even if the $T T R_{\ell}$ does not follow an exponential distribution. The rate cut, which ranges between 0 and 1, describes the fraction of the maximum capacity that is lost during the failure. A rate cut of 1 indicates total failure. All the rate cut information and distribution parameters are given as part of the reliability data of the units. At any point in time there can be a combination of failures occurring in the $m \in M$ units in the network. The combination of failures at any instant is called a discrete state of the integrated site. The set $s=1,2, \ldots,|S|$, is used to refer to such states. The binary vector $y^{s}$ has an element for each of the possible failures of each of the production units in the integrated site. Since a unit can experience more than one type of failure, the vector $y^{s}$ can have more than one element per production unit. This vector contains a 1 for each failure that does not occur as part of state $s$, and a 0 in the position associated with a failure that occurs as part of state $s$. Note that $y^{s}$ is a fixed parameter. The next equation describes the effect of failures on the capacity of the plants in the network:

$p s_{m, s, k 1, k 2} \leq\left(b c_{m}+p c_{m}\right)\left[1-\left(1-y_{\ell}^{s}\right)\left(r c_{\ell}\right)\right] \forall m \in M, \ell \in L_{m}, s \in S, k_{1} \in K_{1}, k_{2} \in K_{2}$

$p s_{m, s, k 1, k 2}$ represents the flow supplied to the $m^{\text {th }}$ production unit during state $s$ and quadrature point $\left(k_{1}, k_{2}\right)$. The parameter $b c_{m}$ is the base processing capacity of unit $m$. The design variable $p c_{m}$ represents extra capacity additions. The parameter $y_{\ell}^{s}$ is the value assigned to vector $y^{s}$ in the position corresponding to $\boldsymbol{e}^{\text {th }}$ failure in the $m^{\text {th }}$ production unit (since $\ell \in L_{m}$ ). Finally, $r c_{\ell}$ is the rate cut corresponding to failure $\ell$. The long term probability of having failure $\ell$ in the integrated site at any instant is given by (Billinton and Allan, 1992): 


$$
p_{\ell}=\frac{M T T R_{\ell}}{M T T R_{\ell}+M T T F_{\ell}}
$$

The long term probability of finding the integrated site in state $s$ is given by:

$$
\operatorname{prob}^{s}=\prod_{\ell: y_{\ell}^{s}=0} p_{\ell} \prod_{\ell: y_{\ell}^{s}=1}\left(1-p_{\ell}\right)
$$

The integrated site is continuously transitioning between discrete states. This behavior is modeled as a continuous time Markov chain where each state $s$ corresponds to a cycle time $t c^{s}$, frequency $f r^{s}$, and mean residence time $m r t^{s}$. As explained in the background section, all these quantities can be calculated from the MTTR and MTTF of the units in the integrated site and can be considered given parameters.

\section{Modeling of intermediate storage}

The flows in and out of the intermediate storage tank after process $j$ are represented by the variables $f_{j, n, s, k 1, k 2}^{I N}$ and $f_{j, n, s, k 1, k 2}^{o U T}$. To represent the difference between these flows, we define the decision variable $\delta_{j, n, k 1, k 2}^{S}=f_{j, n, s, k 1, k 2}^{I N}-f_{j, n, s, k 1, k 2}^{O U T}$. For the sake of simplicity, in most of this section we use the notation $\delta^{S}$, and later use it in full indexed form. The transitions between discrete states $s \in S$ are described by a continuous time Markov process. The bounds on the feasible rate of depletion or replenishment of the inventory $\delta^{S}$ during state $s$ depend on the duration of the state, the amount of material in the tank, and the available storage volume at the beginning of state $s$. Not all of these quantities are deterministic. The duration of $s$ follows a probabilistic distribution determined by the time to repair TTR and time to failure TTF of the units in the integrated site. The material and available space depend not only on the size of the tank, but also on the sequence of states that brought the system to state $s$. Figure 9 shows one possible trajectory for the system. The $x$-axis corresponds to time. The points in this axis are those instants in which the system transitions between states. We will denote these instants epochs. The $y$-axis shows the inventory levels as a function of time or epochs. The slope between epochs $i$ and $i+1$ represents the rate of consumption or replenishment of the inventory during the state $s$ resided in during the interval $t_{i+1}-t_{i}$. This interval is also the 
residence time $r t^{s}$ in state $s$. Note that the states $s \in S$ refer to the discrete states of the continuous time Markov process described by failure and repair events in the integrated site. The amount of materials $X^{i}$ consumed or accumulated during the interval $t_{i+1}-t_{i}$ while the system is in state $s$ is given by,

$X^{i}=\delta^{s(i)} r t^{s(i)} \quad \forall i$, and $s(i)$ corresponding to the state at epoch $i$

$X^{i}$ is a random variable since the residence time $r t^{s(i)}$ in state $s(i)$, that is $t_{i+1}-t_{i}$, depends on the TTR of the failed units, and the TTF of the active units in state $s(i)$. Both, TTR and TTF are described by probabilistic distributions. The inventory level at the time $t(q)$ corresponding to the start of the $q-t h$ epoch, is given by the following equation:

$\operatorname{inv}(t(q))=i n v_{o}+\sum_{i=1}^{q-1} X^{i}=i n v_{0}+\sum_{i=1}^{q-1} \delta^{s(i)} r t^{s(i)}$

Note that such a definition of $\operatorname{inv}(t(q))$ involves a summation of random variables, making $\operatorname{inv}(t(q))$ also a random variable.

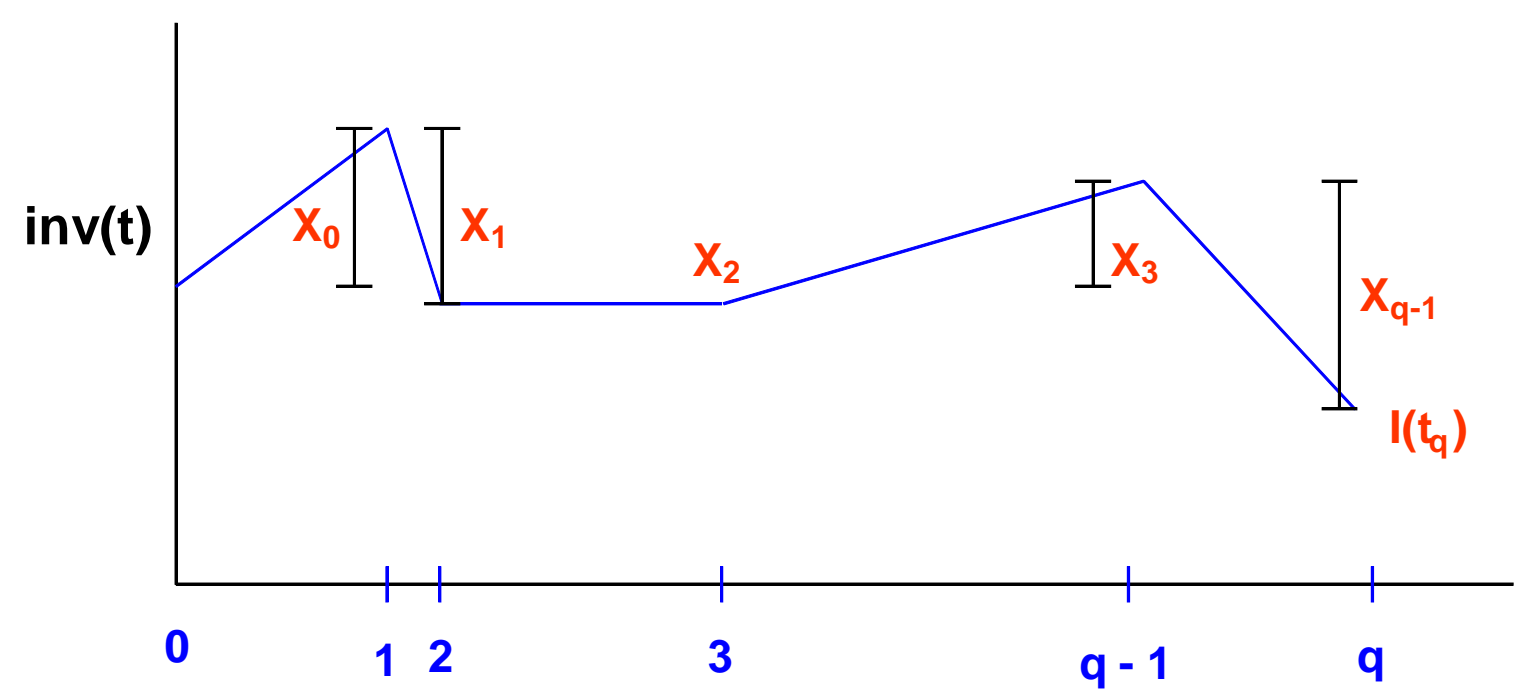

Fig. 9 One possible sequence of states and the corresponding inventory levels in an intermediate tank

We now relate the mean $\overline{i n v}$ and standard deviation $s d$ of the inventory level after a long time of operation $(t(q) \rightarrow \infty)$ to the properties of the states $s \in S$ known "a priori" - 
the mean residence time $m r t^{s}$, frequency of encounters $f r^{s}$, and cycle time $t c^{s}-$ and the values of the decision variables $\delta^{s}$ and $i n v_{0}$.

Since the expectation of a sum of variables is equal to the sum of its expectations (Uspensky, 1937), we calculate the mean inventory level as:

$\overline{i n v}=E(\operatorname{inv}(q))=E\left(i n v_{o}\right)+E\left(\sum_{i=1}^{q-1} \delta^{s(i)} r t^{s(i)}\right)=i n v_{0}+\sum_{i=1}^{q-1} \delta^{s(i)} m r t^{s(i)}$

where $m r t^{s}$ is the mean residence time of state $s$.

The crucial step that follows is changing the summation over epochs $1,2, \ldots, q-1$ into a summation over states $s$. In this work, we are interested in the stationary behavior of the system where $t \rightarrow \infty$. After a long time of operation $t(q) \rightarrow \infty$, each state will be encountered $f r^{s} t$ times. In practice, $t$ is a value long enough that allows the Markov process (of random failures and repairs) to achieve the stationary state. Equations (37) and (38) will show that the expected inventory level $(\overline{i n v})$ is not sensitive to the exact value chosen for $t$. Recall that $f r^{s}$ can be calculated as the probability of finding the state $\operatorname{prob}^{s}$ times the rate of departure from the state. The way in which the frequency $f r^{s}$ is calculated from the reliability information of the units in the integrated site is described in detail in the background section. Using this information, equation (36) becomes:

$\overline{i n v}=i n v_{o}+\sum_{s \in S} \delta^{s} m r t^{s} f r^{s} t$

To make sure that $\overline{i n v}$ remains bounded and kept close to inv as $t(q) \rightarrow \infty$, we add the following constraint:

$\sum_{s \in S} \delta^{s} m r t^{s} f r^{s}=0$

Equations (37) and (38) imply that as $t \rightarrow \infty, \overline{i n v}=i n v_{0}$. These equations are important because they relate the amount of inventory consumed or accumulated in each state $\delta^{s}$ as the plants undergo failure and repair events, to a long term average inventory level. To incorporate the effect of quadrature points, equation (38) is substituted by the following two equations: 


$$
\begin{array}{ll}
\bar{\delta}_{j, n}^{s}=\sum_{k 1 \in K 1} \sum_{k 2 \in K 2} \gamma_{k 1, k 2} \delta_{j, n, k 1, k 2}^{s} & \forall s \in S, j \in J, n \in N \\
\sum_{s \in S} \bar{\delta}_{j, n}^{s} m r t^{s} f r^{s}=0 & \forall j \in J, n \in N
\end{array}
$$

where $k_{1}, k_{2}$ are the indices of the quadrature points of the uncertain parameters (only two parameters are used for consistency with equations (10) - (14), (17), but the model is not limited to this number of parameters) and $\gamma_{k 1, k 2}$ is the probability of the quadrature point $\left(k_{1}, k_{2}\right) . \quad \bar{\delta}_{j, n}^{s}$ is the average rate of consumption or accumulation in the storage tank during state $s$. The inventory level, modeled as a random process in equations (35) - (40), is similar to the well-known model of random walks (Heyman and Sobel, 1982). In our formulation we have to account for the fact that the "walk", or in other words the inventory level, is not unbounded.

We derive the standard deviation $s d$ in an analogous way as the expected inventory level. Refer to equation (35) and recall that $i n v(t)$ is a random variable, and that it includes a summation where the residence time in each state $r t^{s}$ appears. We use the following definition for the variance of a sum of random variables (Uspensky, 1937) :

$$
\operatorname{VAR}\left(\sum_{i \in I} Y_{i}\right)=\sum_{i \in I} \operatorname{VAR}\left(Y_{i}\right)+2 \sum_{i<j} \operatorname{COVAR}\left(Y_{i}, Y_{j}\right)
$$

The variables $Y_{i}$ correspond to $i n v_{o}+\sum_{i=1}^{q-1} \bar{\delta}^{s(i)} r t^{s(i)}$. Since the residence times in each state $\left(r t^{s}\right)$ are independent, the COVAR term in equation (41) is equal to zero, yielding,

$$
\operatorname{VAR}(\mathrm{inv})=\sum_{i=1}^{q-1} \operatorname{VAR}\left(\bar{\delta}^{s(i)} r t^{s(i)}\right)=\sum_{i=1}^{q-1} \bar{\delta}^{s(i)^{2}} \operatorname{VAR}\left(r t^{s(i)}\right)
$$

We can change the summation over epochs $1,2, \ldots, q-1$ into a summation over states $s$ as in equation (37), so that equation (42) becomes:

$$
\operatorname{VAR}(\mathrm{inv})=\sum_{s \in S} f r^{s} t \bar{\delta}^{s^{2}} \operatorname{VAR}\left(r t^{s}\right)
$$

It then follows that the standard deviation is:

$$
s d=s d(\operatorname{inv}(q))=\sqrt{\operatorname{VAR}(\operatorname{inv})}=\sqrt{\sum_{s \in S} f r^{s} t \bar{\delta}^{s^{2}} V A R\left(r t^{s}\right)} .
$$

In order to keep the optimization model linear, we use the following overestimation of $s d$, 
$s d=\sqrt{\sum_{s \in S} f r^{s} t \bar{\delta}^{s^{2}} V A R\left(r t^{s}\right)} \leq \sum_{s \in S} \sqrt{f r^{s} t \mid}\left|\bar{\delta}^{s}\right| \sqrt{\operatorname{VAR}\left(r t^{s}\right)}=\tilde{s d}$,

where $\sqrt{\operatorname{VAR}\left(r t^{s}\right)}=S D^{s}$. SD $D^{s}$ can be determined from the reliability data of the integrated site. In the case where the $T T F$ and TTR follow exponential distributions, the variance of the residence time $r t^{s}$ is equal to its mean; that is, $S D^{s}=\sqrt{m r t^{s}}$. In the second numerical example presented in this paper, we use the simplification $S D^{s}=\sqrt{m r t^{s}}$ even though the TTR follows a normal distribution. We are aware that this is not the exact variance for a normal distribution, but given that we are only using approximate formulas for the variance and standard deviation of the residence time (i.e., equation 45) we assume this simplification to be a first approximation to the behavior of the system.

Recovering the full indices of the decision variables, the equation for determining an overestimation of the standard deviation of the inventory level (inv) as $t \rightarrow \infty$ is given as follows:

$s \tilde{d}_{j, n}=\sum_{s \in S} S D^{s}\left|\bar{\delta}_{j, n}^{s}\right| \sqrt{f r^{s} t} \quad \forall j \in J, n \in N$

Notice that there is a term $t$ in equation (46) that stands for a long time of operation $(t \rightarrow \infty)$. In practice we set $t$ equal to the time we expect the integrated site to operate continuously. A good estimate for $t$ is the time between scheduled maintenance, or any other major operation interruptions. In the examples presented in a later section, the time $t$ was set equal to one year of operation. To make sure that the probabilistic distribution of the inventory after a long time operation $(\operatorname{inv}(t), t \rightarrow \infty)$ falls within the physical bounds of the holding tank, we use the following two equations:

$$
\begin{array}{lc}
\overline{i n v}_{j, n}+\tau_{u p} \tilde{s d}_{j, n} \leq v_{j, n} & \forall j \in J, n \in N \\
\overline{i n v}_{j, n}-\tau_{l o} \tilde{s d_{j, n} \geq 0} & \forall j \in J, n \in N
\end{array}
$$

where $v_{j, n}$ is the storage capacity of product $n$ in plant $j$ and $\tau_{u p}$ and $\tau_{l o}$ are parameters that truncate the area under the probability distribution of the inventory level (inv). If we 
assume that the inventory level follows a normal distribution we can set $\tau_{u p}=\tau_{l o}=4$, which leads to a $99.99 \%$ probability of the distribution function.

In equation (46), we are using $\bar{\delta}^{s}$ that corresponds to the average of $\delta_{k 1, k 2}^{s}$ over all collocation points $k_{1}$ and $k_{2}$, as obtained in equation (39). This average rate of inventory consumption/replenishment $\bar{\delta}^{s}$ could have a moderate value as a result of averaging large negative (consumption) rates $\delta_{k 1, k 2}^{s}$ in some collocation points and large positive (replenishment) rates in others. For example, $\bar{\delta}^{s}$ could be 5 units/hour for a 100 unit tank, as a result of averaging $\delta_{k 1, k 2}^{s}=-9,990$ and $\delta_{k 1^{\prime}, k 2^{\prime}}^{s}=10,000$ units/hour in two equally probable collocation points. In order to constrain the value of $\delta_{k 1, k 2}^{s}$ at each collocation point, we add constraints (49) and (50).

$$
\begin{array}{lc}
\overline{i n v}+\delta_{j, n, k 1, k 2}^{s} m r t^{s} \leq v_{j, n} & \forall j \in J, n \in N, s \in S, k_{1} \in K_{1}, k_{2} \in K_{2} \\
\overline{i n v}-\delta_{j, n, k 1, k 2}^{s} m r t^{s} \geq 0 & \forall j \in J, n \in N, s \in S, k_{1} \in K_{1}, k_{2} \in K_{2}
\end{array}
$$

Finally, the intermediate storage volume is bounded by its maximum capacity:

$$
0 \leq v_{j, n} \leq v_{\mathrm{j}, \mathrm{n}}^{\mathrm{UB}} \quad \forall j \in J, n \in N
$$

\section{Cost function}

In this work, we use the linear cost function (52) for minimizing capital investment required by the integrated site design. Installing a processing unit with its base capacity has a fixed cost, and a variable cost for the extra capacity addition. The cost associated with intermediate storage is assumed to vary linearly with the size of the tank.

$$
\min \text { cap }=\sum_{j \in J} \sum_{m \in M_{j}} \sum_{n \in N}\left(\beta_{m}^{f i x} z_{m}+\beta_{m}^{\mathrm{var}} p c_{m}+\beta_{j, n}^{\mathrm{tank}} v_{j, n}\right)
$$

In the above objective function, $\beta_{m}^{f i x}$ is the fixed cost for including unit $m$ in the integrated site and $\beta_{m}^{\mathrm{var}}$ is the variable cost associated extra capacity addition $\left(p c_{m}\right)$. $\beta_{j, n}^{\text {tank }}$ is the cost related to the size of the intermediate tank for product $n$ after plant $j$. Since the problem is a bi-criterion optimization, the cost function (52) is minimized while the expected stochastic flexibility in (17) is maximized. 


\section{Superstructure optimization}

In this work, we assume that a superstructure of design alternatives is specified for the integrated site. The superstructure consists of the network of processes in the site, where each process is represented by the building block in Figure 8. In order to model the selection of the optimal design embedded in the superstructure, we define the binary variables $z_{m}$ that take a value of 1 if the $m^{\text {th }}$ production unit is included in the integrated site and 0 otherwise. This variable is used in following constraint,

$$
p s_{m, s, k 1, k 2} \leq M U z_{m} \quad \forall j \in J, m \in M_{j}, s \in S, k_{1} \in K_{1}, k_{2} \in K_{2}
$$

where $M U$ is a valid upper bound for the flow processed by the $m^{\text {th }}$ production unit. We also use the variables $z_{m}$ in the logic cuts (Hooker et al., 1994) given by equations (54) and (55).

$$
\begin{aligned}
& v_{j, n} \leq v_{j, n}^{U P} \sum_{m \in M_{j}} z_{m} \quad \forall j \in J, n \in N \\
& \sum_{m} \mathbf{a}_{m} z_{m} \leq \mathbf{b}
\end{aligned}
$$

Equation (54) allows storage $v_{j, n}$ to be built only for those plants $j$ that are part of the integrated site. The units $m \in M_{j}$ are the parallel units proposed for plant $j$ in the superstructure, and the term $\sum_{m \in M_{j}} z_{m}$ will be zero if none of these units is part of the optimal design (i.e. plant $j$ is not part of the integrated site). Equation (55) is a set of linear inequalities that expresses logical relations on the network topology of the integrated site in terms of the variables $z_{m}$.

The complete optimization model is defined by equations (17)-(23), (26)-(31), (39), (40), (46)-(55), plus constraints (56) and (57) which are introduced in a later section of the paper. This formulation involves the integration of a state-space model and a superstructure optimization approach. Most of the problem variables and constraints are defined for each state and collocation point, therefore, the dimensionality of the optimization problem grows exponentially with the number of possible failures in the superstructure and the number of uncertain parameters. Table 1 includes a summary of all the decision variables included in this problem. Examples 1 and 2 in this paper illustrate 
the problem sizes that results from applying the proposed formulation to specific integrated sites.

Table 1 Decision variables in the optimization model

Continuous design variables

Process capacity, $p c_{m}$

Storage tank capacity $\quad v_{j, n}$

Average storage level $\quad \overline{i n v}_{j, n}$

Standard deviation of levels of storage $\quad s d_{j, n}$

Continuous operation variables

Flow among plants $\quad$ flow $_{j^{\prime}, j, n, s, k 1, k 2}$

Flow from production units $\quad f_{j, n, s, k 1, k 2}^{P}$

Flow into storage tanks $\quad f_{j, n, s, k 1, k 2}^{I N}$

Flow out of storage tanks $\quad f_{j, n, s, k 1, k 2}^{\text {OUT }}$

Flow that bypasses the storage tank $\quad f_{j, n, s, k 1, k 2}^{B}$

Accumulation rate in storage $\quad \delta_{j, n, k 1, k 2}^{S}$

Average accumulation rate per state $\quad \bar{\delta}_{j, n}^{S}$

Absolute value of accumulation rate

$\left|\bar{\delta}_{j, n}^{S}\right|$

\begin{tabular}{lc}
\hline Auxiliary continuous variables & \\
\hline Slack variable & $u_{k 1, k 2}^{s}$ \\
\hline Discrete variables & $w_{k 1, k 2}^{s}$ \\
\hline Feasibility variable & $z_{m}$ \\
Unit selection variable & \\
\hline
\end{tabular}

The integration of the state-space model and the superstructure optimization approach presents challenges that are not found in standard superstructure optimization problems. Each possible flowsheet corresponds to a different state space; if unit $m$ is not part of the 
flowsheet then the failure modes that occur in this unit should not be considered when deriving the state-space from all the combinations of possible failures in the flowsheet. For instance, a flowsheet with 4 units, where each unit can be either UP or DOWN, will have a state-space of cardinality 16 . A flowsheet with 10 units will have a state-space of cardinality 1024. Furthermore, the probability of finding any state in the flowsheet with 4 units will be different (i.e., larger) that finding any state in the flowsheet with 10 units. The probability of finding state $s$ is $p r o b_{s}$ in the objective function (17). If $p r o b_{s}$ becomes a variable -as it appears to be the case in a superstructure optimization problemequation (17) becomes bilinear and nonconvex, destroying the MILP structure of the problem. Our objective in this part of the paper is to propose an approach where the probabilities of finding each state $p r o b_{s}$ and all the other reliability indicators, $m r t^{s}, f r^{s}, t c^{s}$, are kept as problem parameters, even if we use a superstructure approach. In the remainder of this section we present our result, describe it qualitatively, and then provide rigorous mathematical propositions that validate it.

Our result is the following: we solve the problem defined by equations (17)-(23), (26)(31), (39), (40), (46)-(55), plus constraints (56) and (57) defined below, for all the states $s \in S$ resulting from all the potential production units in the superstructure and obtain an optimal selection of processes that we call $\bar{M}$, as will be rigorously proved in the next section. We claim that this solution (with the complete state space $S$ ) is equivalent to that obtained by solving the problem starting with just the optimal selection $\bar{M}$. It should be clear that this result is not obvious. The reasons are that when we solve the problem for the state-space derived from the units in the superstructure we are considering failure modes and repair actions for units that do not exist in the optimal flowsheet; also, we are using reliability data $p_{s}, m r t^{s}, f r^{s}, t c^{s}$ of a system that corresponds to all the units in the superstructure. These data are different form the data corresponding only to the optimal flowsheet.

$$
\begin{array}{ll}
\bar{\delta}_{j}^{s} \leq \bar{\delta}_{j}^{s^{\prime}}+M \sum_{m \in M} \sum_{\ell \in L_{m}} z_{m} \sigma_{\ell}^{s, s^{\prime}} & \forall j \in J, s \in S, s^{\prime} \in S, s>s^{\prime} \\
\bar{\delta}_{j}^{s} \geq \bar{\delta}_{j}^{s^{\prime}}-M \sum_{m \in M} \sum_{\ell \in L_{m}} z_{m} \sigma_{\ell}^{s, s^{\prime}} & \forall j \in J, s \in S, s^{\prime} \in S, s>s^{\prime}
\end{array}
$$


Before proceeding with the qualitative explanation of the result we describe equations (56) and (57). Recall that $\bar{\delta}_{j}^{s}$ is the average rate of consumption or depletion of material in the intermediate storage tank after plant $j$ during state $s$. In (56) and (57) $M$ is a valid upper bound (a big-M), and $\sigma_{\ell}^{s, s}$ is a problem parameter that can be derived form the vector $y_{\ell}^{s}$. The vector $y_{\ell}^{s}$ is a fixed parameter defined in a previous section to be 1 if failure $\ell$ does not occur as part of state $s$, and 0 otherwise. Let the parameter $\sigma_{\ell}^{s, s}$ be defined as follows.

$\sigma_{\ell}^{s, s}=\min \left\{y_{\ell}^{s}-y_{\ell}^{s^{\prime}},\left(1-y_{\ell}^{s}\right)-\left(1-y_{\ell}^{s^{\prime}}\right)\right\} \quad \forall \ell \in L, s \in S, s^{\prime} \in S, s>s^{\prime}$

In equation (58) $y_{\ell}^{s}$ is set to one if states $s$ and $s^{\prime}$ are distinguishable with respect to failure $\ell$; that is, if the failure occurs in one state and not in the other. Equations (56) and (57) set the values of $\bar{\delta}_{j}^{s}$ and $\bar{\delta}_{j}^{s^{\prime}}$ equal for the pairs $s$ and $s^{\prime}$ that are indistinguishable with respect to the failures of the $m$ units installed.

To illustrate the main idea behind the result mentioned before, assume there is a superstructure that consists of 3 units. Each of these units can be in an UP or DOWN state. Figure 10 shows the different state-spaces that result from different selections of units from the superstructure.

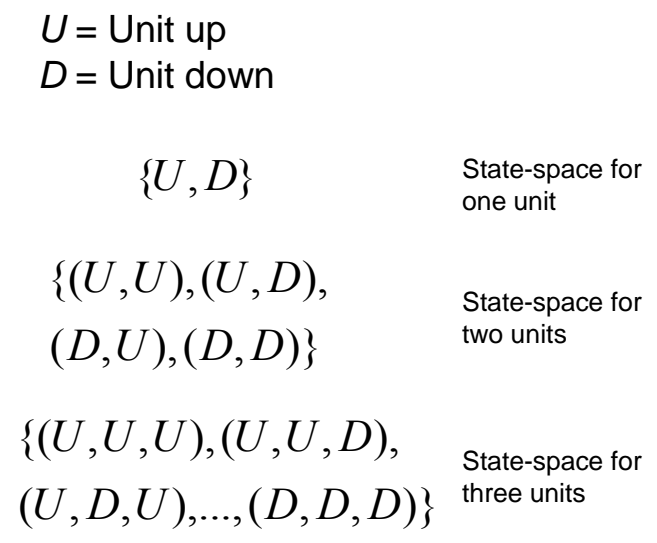

$$
\text { one unit }
$$

State-space for two units

State-space for three units

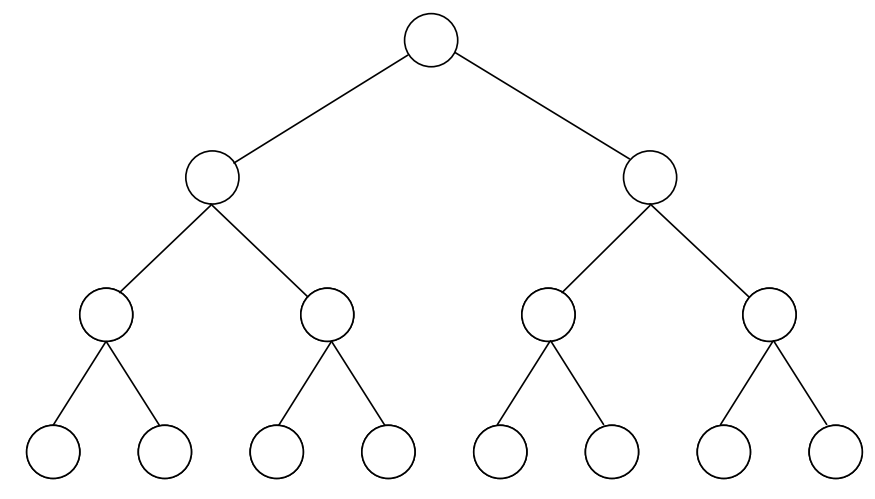

Fig. 10 State-space representation as a function of the number of units

Our result says that we can optimize over the state-space in the last level of the tree ( 8 states), even if the optimal flowsheet contains only one or two units. Figure 11a shows that if only one unit is installed, the four states in the last level of the tree that are derived 
from the same node in the one unit state-space are identical in terms of feasibility/infeasibility. This figure also shows that the sum of the probabilities of the four states in the last level of the tree is equal to the probability of the state from which they are derived in the one unit state-space. Figure $11 \mathrm{~b}$ shows the equivalent result for the case where two units are installed.

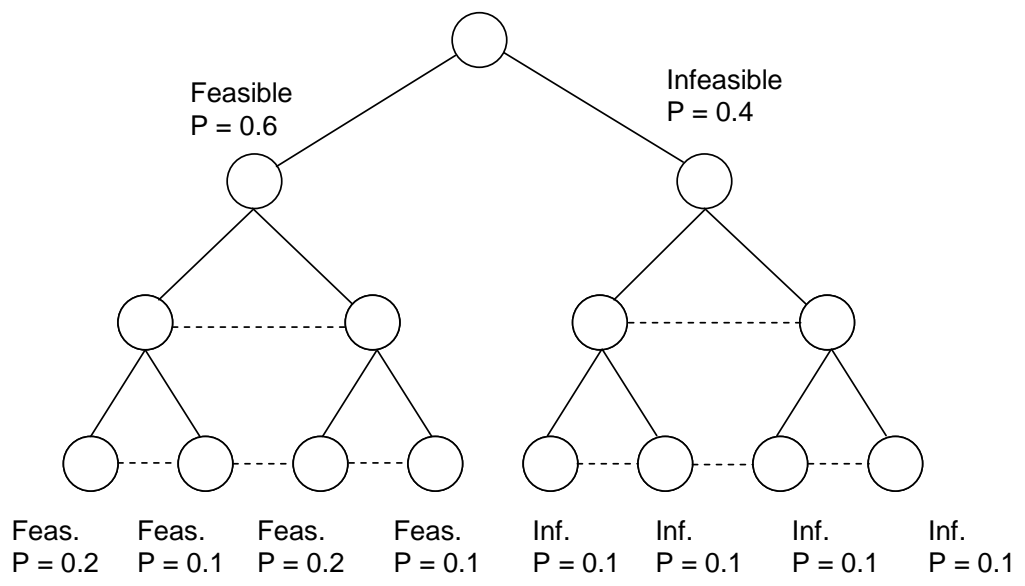

Fig. 11a Optimization of a flowsheet with one unit using the three unit state-space

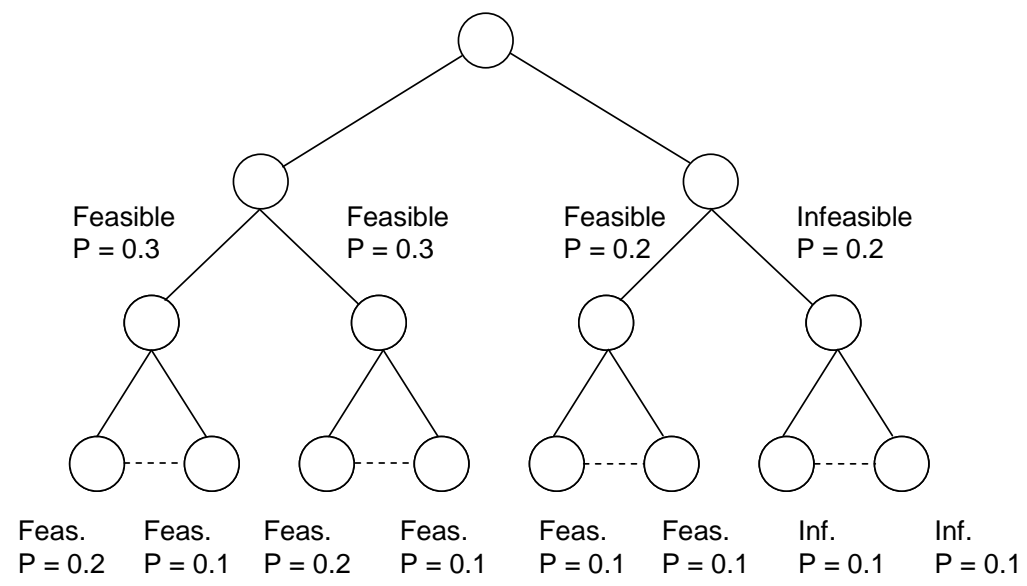

Fig. 11b Optimization of a flowsheet with two units using the three unit state-space

Note that the relationships of common feasibility/infeasibility and of the summation of probabilities appear in our problem without adding any extra constraints except for equations (56) and (57). In the case illustrated in Figure 11a, equations (56) and (57) set the rate of consumption/replenishment of inventory $\bar{\delta}^{s}$ equal for each of the groups of 
four states linked by the doted lines. The reason that explains why these constraints are needed for the variable $\bar{\delta}^{s}$ but not for any of the other variables in the model can be expressed in a simplified way as follows. The flow variables, $p s, f$, flow, are zero for those units that are excluded from the optimal flowsheet by equation (53), regardless of whether the unit is described as UP or DOWN in the states in the last level of Figure 11a. However, the variable $\bar{\delta}^{s}$ appears in equations (40) and (46) where the duration and the frequency of appearance of each state are involved. If we use the state-space in the last level of the tree in Figure 11a, each of the nodes corresponds to a state with a different duration and frequency so that different feasible rates of consumption/depletion of inventory can be chosen for each of the states in this level. Equations (56) and (57) prevent this from occurring. This discussion is formalized in the next subsection.

\section{Properties of the superstructure optimization model}

To prove the above claim on the superstructure optimization, we review some nomenclature and introduce some new sets. Let an integrated site have $j \in J$ plants, where each of them can have $m \in M_{j}$ parallel production units. The set $M=\bigcup_{j} M_{j}$ contains all the candidate production units in the integrated site. Each $m \in M$ is subject to $\ell \in L_{m}$ potential failures. The set $L=\bigcup_{m} L_{m}$ contains all the possible failures of all the candidate production units in the superstructure of the integrated site. Recall that each discrete state is a particular combination of failures occurring simultaneously. The state space $S$ contains all the states resulting from the combination of the $\ell \in L$ failures that can occur in any of the $m \in M$ processing units.

Remark There is a one to one correspondence between a set $M$ and a set $L$. Also each set $L$ generates one particular state space $S$.

Suppose we solve the optimization problem in equations (17)-(23), (26)-(31), (39), (40), (46)-(57) and find that the optimal solution involves only a subset of the units postulated in the superstructure; we represent this subset as $\bar{M} \subset M$. This means $z_{m}=1, m \in \bar{M}$ and $z_{m}=0, m \in M \backslash \bar{M}$. The possible failures relevant to the set $\bar{M}$ are contained in set 
$\bar{L}=\bigcup_{m \in \bar{M}} L_{m}$

The reduced set $\bar{S}$ is the space state corresponding to all the possible combinations of failures in $\bar{L}$, that is, those failures relevant to the units included in the optimal design. So far we have defined $M, L, S$, for the units, possible failures, and states of the superstructure, and the reduced sets $\bar{M}, \bar{L}, \bar{S}$, for the units, possible failures and states of the optimal design.

Each state $s \in S$ corresponds to a vector $y^{s} \in\{0,1\}^{|L|}$. A zero in the $\boldsymbol{e}^{\text {th }}$ position of this vector indicates that failure $\ell \in L$ occurs while the integrated site is in state $s \in S$. In the particular case of one total failure per unit, a zero in this vector also indicates that a unit is down and a value of 1 indicates that a unit is up. Similarly, each state $\bar{s} \in \bar{S}$ corresponds to a reduced vector $y^{\bar{s}} \in\{0,1\}^{|\bar{L}|}$. Recall that $\bar{L} \subset L$, so $y^{s}$ has larger dimensionality than $y^{\bar{s}}$. Each vector $y^{s} \in\{0,1\}^{|L|}$ can be projected onto the space $\{0,1\}^{|\bar{L}|}$. We denote this projected vector as $y_{p r o j|\bar{L}|}^{s}$. Each $y_{p r o j \mid}^{s}|\bar{L}|$ is identical to a reduced vector $y^{\bar{s}} \in\{0,1\}^{|\bar{L}|}$. Some vectors $y^{s} \in\{0,1\}^{|L|}$ will share the same projection $y_{\text {proj }|\bar{L}|}^{s}$ (or $\left.y^{\bar{s}} \in\{0,1\}^{|\bar{L}|}\right)$.

To clarify the notation, suppose we have a one-process system with two potential parallel units. In this case: $J=\left\{j_{1}\right\}, M_{j 1}=\left\{m_{1}, m_{2}\right\}$, and $M=\left\{m_{1}, m_{2}\right\}$. Units $m_{1}$ and $m_{2}$ are subject to one random total failure each, so $L_{m 1}=\left\{\boldsymbol{\ell}_{1}\right\}, L_{m 2}=\left\{\boldsymbol{\ell}_{2}\right\}$, and $L=\left\{\boldsymbol{\ell}_{1}, \boldsymbol{\ell}_{2}\right\}$. The state space $S$ is constructed from all the possible combinations of failures in the system. Thus, $S=\left\{s_{1}, s_{2}, s_{3}, s_{4}\right\} ; s_{1}$ corresponds to NO failure, $s_{2}$ to failure $\ell_{1}, s_{3}$ to failure $\ell_{2}$, and $s_{4}$ to failures $\ell_{1}$ AND $\ell_{2}$ as shown in Table 2 . 
Table 2 Discrete states in a superstructure with two units.

\begin{tabular}{ccc}
\hline State (s) & Unit 1 & Unit 2 \\
\hline 1 & UP & UP \\
2 & DOWN & UP \\
3 & UP & DOWN \\
4 & DOWN & DOWN \\
\hline
\end{tabular}

Each state $s \in S$ corresponds to one vector $y^{s}$, of length 2 . Table 3 shows the vectors $y^{s}$ that correspond to the states in Table 1.

Table 3 Vectors $y^{s}$ corresponding to the states in a superstructure with two units.

\begin{tabular}{cc}
\hline State (s) & $\mathbf{y}^{\mathbf{s}}$ \\
\hline 1 & {$\left[\begin{array}{ll}1 & 1\end{array}\right]$} \\
2 & {$\left[\begin{array}{ll}0 & 1\end{array}\right]$} \\
3 & {$\left[\begin{array}{ll}1 & 0\end{array}\right]$} \\
4 & {$\left[\begin{array}{ll}0 & 0\end{array}\right]$} \\
\hline
\end{tabular}

If we solve the optimal design problem and find that only $m_{1}$ is included in the optimal solution, we have the following reduced sets: $\bar{M}=\left\{m_{1}\right\}, \bar{L}=\left\{\boldsymbol{\ell}_{1}\right\}$ and $\bar{S}=\left\{\overline{s_{1}}, \overline{s_{2}}\right\}$. Table 4 contains the reduced states $\bar{s} \in \bar{S}$ corresponding to the units in the optimal design, and Table 5 shows the vector $\bar{y}^{s}$ corresponding to the states in Table 4.

Table 4 Discrete states in an optimal design with one unit.

\begin{tabular}{cc}
\hline State $(\overline{\mathbf{s}})$ & Unit $\mathbf{1}$ \\
\hline$\overline{1}$ & UP \\
$\overline{2}$ & DOWN \\
\hline
\end{tabular}


Table 5 Vectors $\bar{y}^{s}$ corresponding to the states an optimal design with one unit.

\begin{tabular}{cc}
\hline State $(\bar{s})$ & $\bar{y}^{s}$ \\
\hline$\overline{1}$ & {$[1]$} \\
$\overline{2}$ & {$[0]$}
\end{tabular}

The projection of $y^{s 1}$ and $y^{s 3}$ in Table 3 onto the space $\{0,1\}^{1}$ is equal to $y^{\overline{s 1}}$ in Table 5 , and the projection of $y^{s 2}$ and $y^{s 4}$ in Table 3 onto the space $\{0,1\}^{1}$ is equal to $y^{\overline{s 2}}$ in Table 5. We are interested in grouping those states $s \in S$ in the superstructure consisting of $m \in M$ units that share the same projection onto the space $\bar{s} \in \bar{S}$ that takes into account only the optimal selection of units $m \in \bar{M}$. As mentioned before, states $s_{1}, s_{3}$, in the superstructure (Table 2) share the same projection $\overline{s_{1}}$ in the state space of the optimal units (Table 4). We group $s_{1}, s_{3}$ by creating a new projected set $S_{\overline{s 1}}=\left\{s_{1}, s_{3}\right\}$. The rationale behind the notation is that $S_{\overline{s 1}}$ is the subset of $S$ whose states share the projection $\overline{s_{1}} \in \bar{S}$. Likewise, states $s_{2}, s_{4}$ in the superstructure (Table 2) share the same projection $\overline{s_{2}}$ in the state space of the optimal units (Table 4). We group $s_{2}, s_{4}$ by creating the new projected set $S_{\overline{s 2}}=\left\{s_{2}, S_{4}\right\}$.

In general we define the projected set $S_{-}$, as follows:

$S_{-}=\left\{s \mid y_{p r o j|\bar{L}|}^{s}=y^{\bar{s}}\right\} \quad, \bar{s} \in \bar{S}$

\section{Proposition 1}

Let $w^{s}$ with $s \in S$ correspond to the variables in the objective (17), where the indices $k_{1}, k_{2}$ have been omitted for simplicity. Let $S$ be the state space generated by all combinations of failures $\ell \in L$ that occur in all units $m \in M$ in the superstructure. $w^{s}=1$ indicates that the operation of units $m \in M$ is feasible under the combination of possible failures $\ell \in L$ that defines the state $s \in S ; w^{s}=0$ means that the operation is infeasible.

Let $z_{m}=0 \quad \forall m \in M \backslash \bar{M}$. Then, 
$w^{s}=w^{\bar{s}} \quad \forall s \in S_{-}, \bar{s} \in \bar{S}$

This proposition implies that feasible operation of the integrated site depends only on the failed/active status of the units selected from the superstructure. Therefore, all discrete states that differ only on the failed/active status of the units not selected are identical in terms of feasible or infeasible operation. The proof of this proposition can be found in the appendix.

\section{Proposition 2}

The probability $\left(\right.$ prob $^{\bar{s}}$ ) of finding state $\bar{s} \in \bar{S}$ is equivalent to the summation over all the probabilities $\left(\right.$ prob $\left.^{s}\right)$ of finding the states $s \in S_{-}$that share the same projection onto the space $\bar{S}$.

$\operatorname{prob}^{\bar{s}}=\sum_{s \in S_{\bar{s}}} \operatorname{prob}^{s} \quad \forall \bar{s} \in \bar{S}$

The proof of this proposition can be found in the appendix of this paper.

\section{Proposition 3}

Let $E(S F)^{*}$ be a Pareto-optimal solution found using the set $S$ in equations (17)-(23), (26)-(31), (39), (40), (46)-(57), and $\overline{E(S F)}^{*}$ be the Pareto-optimal solution found using the reduced set $\bar{S}$. Then, $E(S F)^{*}=\overline{E(S F)}^{*}$

This proposition is a consequence of Propositions 1 and 2; its proof can be found in the appendix.

Proposition 3 proves the claim that solving the problem given by equations (17)-(23), (26)-(31), (39), (40), (46)-(57) using the space state resulting from all the units postulated in the superstructure, is equivalent to optimizing over the state space defined only for the optimal selection of units.

Note that the propositions in this section can be easily extended to include the effect of quadrature points $\left(k_{1}, k_{2}\right)$.

\section{Example 1}

The following example is based on a case study shown in previous work concerned with evaluating (not optimizing) the $\mathrm{E}(\mathrm{SF})$ of a network of production processes (Straub \& Grossmann, 1990). The example of the chemical complex has been 
modified to include intermediate storage. The objectives are to maximize the expected stochastic flexibility $\mathrm{E}(\mathrm{SF})$ and to minimize the cost of installing parallel units, intermediate storage, and increasing production capacity. The superstructure for the integrated site is shown in Figure 10. This proposed integrated site has three potential plants. Plant 1 transforms A to B and plant 2 transforms B to C. Plant 3 transforms A directly into $\mathrm{C}$. There are two potential parallel units for plant 1 (units $l_{I}$ and $l_{I I}$ ), one for plant 2 (unit 2), and one for plant 3 (unit 3). All three plants have the potential addition of storage.

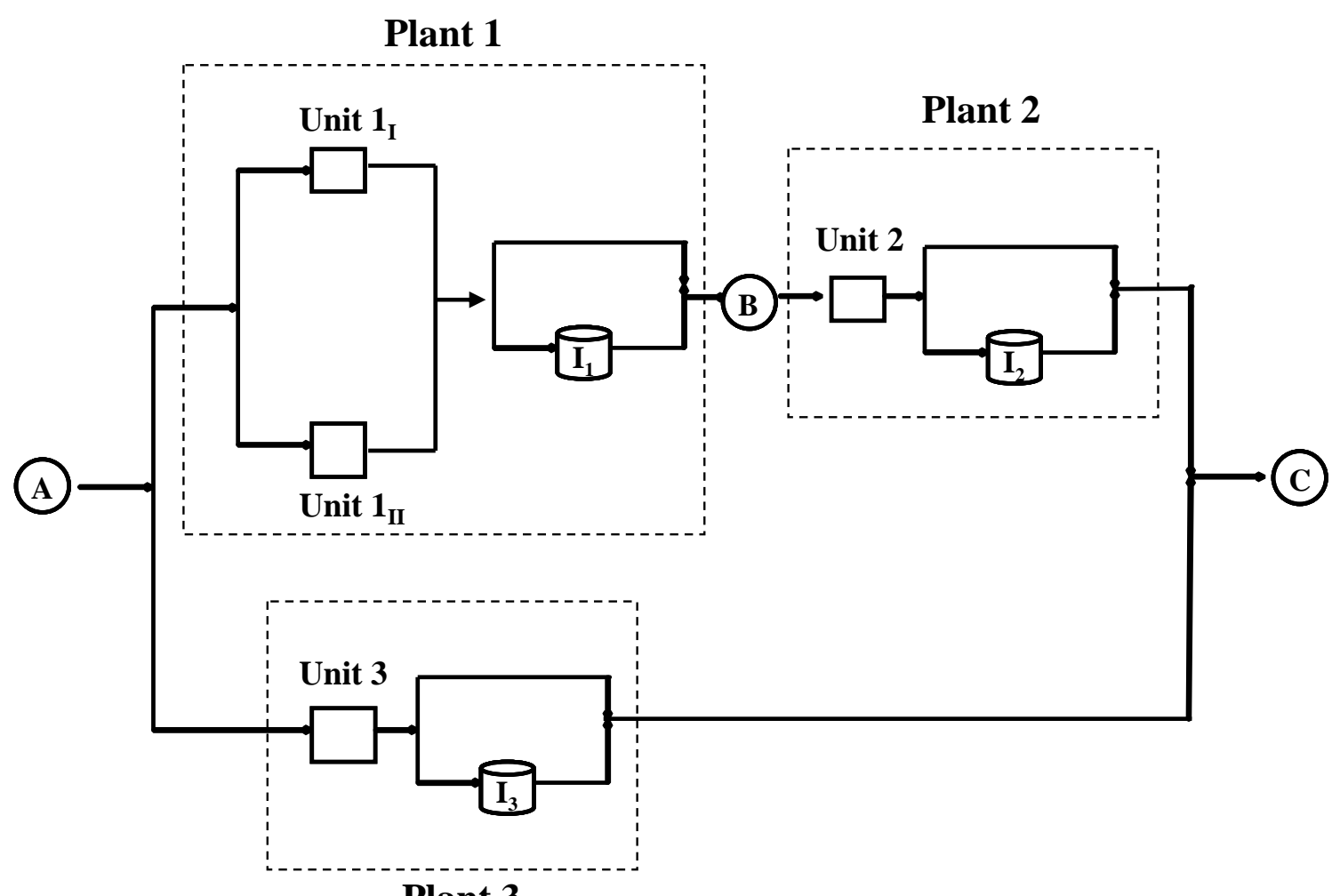

Plant 3

Fig. 10 Integrated Site for the production of $C$ from $A$.

Table 6 contains the data required to solve the problem using equations (17)-(23), (26)(31), (39), (40), (46)-(57). The base capacity provided in this table represents the minimum capacity that each unit will have if it installed. This is a parameter considered fixed in a previous design step. In an industrial setting this might correspond to a design stage where continuous and discrete uncertainties are not considered. In Example 1, we set the base capacities to the values given by Straub \& Grossmann (1990). Note that the 
fixed cost of installing a unit considers this base capacity, so that only additional capacity has an extra variable cost.

There is only one total failure per unit in the integrated site, so that the number of failures and number of units is equal. The uncertain parameters $\theta_{1}$ and $\theta_{2}$ are the supply of $A$ and demand of $\mathrm{C}$. We discretize each of them using 5 collocation points. In order to test the effect of the number of collocation points on the solution to the problem, we also solved this example with 10 quadrature points and found that there is only a slight difference in the optimal solution with a significant increase in solution time. Therefore, we only report the results of using 5 collocation points. The sets $J, M, N, S, K_{1}$ and $K_{2}$ used in the formulation are described explicitly below:

$$
\begin{aligned}
& J=\{1,2,3\}, \\
& M_{1}=\left\{1_{I}, 1_{I I}\right\}, M_{2}=\{2\}, M_{3}=\{3\}, \\
& M=\left\{1_{I}, 1_{I I}, 2,3\right\}, \\
& L_{1 I}=\left\{1_{I}\right\}, L_{1 I I}=\left\{1_{I I}\right\}, L_{2}=\{2\}, L_{3}=\{3\}, \\
& L=\left\{1_{I}, 1_{I I}, 2,3\right\}, \\
& S=\{1,2, \ldots, 16\}, \\
& K_{1}=\{1,2,3,4,5\}, K_{2}=\{1,2,3,4,5\},
\end{aligned}
$$

\section{Table 6 Data for Example 1}

\section{Supply of A}

$$
\text { [10 } 0^{3} \text { ton / day] }
$$$$
\text { Mean }=12
$$$$
\operatorname{Std}=1
$$

Demand of C

[ $10^{3}$ ton / day $]$

$$
\begin{array}{cc}
\text { Mean }=7 & \text { Stand. Dev }=1 \\
\text { Unit } 1_{I} & \text { Unit } 1_{I I}
\end{array}
$$$$
\text { Unit } 2
$$$$
\text { Unit } 3
$$

Probability of operation

0.95

0.95

0.92

0.87

Mass balance coefficient

$$
\alpha_{1 \mathrm{I}}=0.92
$$

$\alpha_{1 \mathrm{II}}=0.92$

$\alpha_{2}=0.85$

$\alpha_{3}=0.75$

Base capacity

$\left[10^{3}\right.$ ton / day $]$

Mean time to repair [day]

Mean time to failure

[day]

\section{5}

0.25

4.75
5

0.25

4.75
7

0.25

0.25

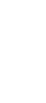

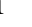


Recall that each of the 16 states is described by a vector $y^{s} \in\{0,1\}^{|L|}$, where a 0 in the $\boldsymbol{\ell}-t$ th position means that failure $\boldsymbol{\ell}$ is present when the integrated site is in state $s$. Table 7 describes each state in terms of its $y^{s}$ vector. For instance, state 1 corresponds to no failure in the integrated site since $y_{\ell}^{1}=1 \forall \ell \in L$.

Table 7 Discrete states for integrated site

\begin{tabular}{|c|c|c|c|c|c|}
\hline & $y_{1 I}^{s}$ & $y_{1 I I}^{s}$ & $y_{2}^{s}$ & $y_{3}^{s}$ & $\operatorname{prob}^{s}$ \\
\hline State 1 & 1 & 1 & 1 & 1 & 0.722 \\
\hline State 2 & 1 & 1 & 1 & 0 & 0.108 \\
\hline State 3 & 1 & 1 & 0 & 1 & 0.063 \\
\hline State 4 & 1 & 0 & 1 & 1 & 0.038 \\
\hline State 5 & 0 & 1 & 1 & 1 & 0.038 \\
\hline State 6 & 1 & 1 & 0 & 0 & 0.009 \\
\hline State 7 & 1 & 0 & 1 & 0 & 0.006 \\
\hline State 8 & 1 & 0 & 0 & 1 & 0.003 \\
\hline State 9 & 0 & 1 & 1 & 0 & 0.006 \\
\hline State 10 & 0 & 1 & 0 & 1 & 0.003 \\
\hline State 11 & 0 & 0 & 1 & 1 & 0.002 \\
\hline State 12 & 1 & 0 & 0 & 0 & $5 e-4$ \\
\hline State 13 & 0 & 1 & 0 & 0 & $5 e-4$ \\
\hline State 14 & 0 & 0 & 1 & 0 & $3 e-4$ \\
\hline State 15 & 0 & 0 & 0 & 1 & $1 \mathrm{e}-4$ \\
\hline State 16 & 0 & 0 & 0 & 0 & $3 e-5$ \\
\hline
\end{tabular}


We assume a normal distribution for both uncertain parameters, $\theta_{1}$ (supply) and $\theta_{2}$ (demand), and bound them at their mean value $+/$ - four standard deviations. We then use a five point Gaussian Quadrature scheme to obtain the quadrature points in Table 8, which are derived from the roots of the Legendre polynomial (Carnahan et al., 1969).

Table 8 Five point Gaussian quadrature for demand and supply

\begin{tabular}{|c|c|c|c|c|c|c|c|}
\hline & $\begin{array}{l}\text { Lower } \\
\text { Bound }\end{array}$ & $k_{1} / k_{2}=1$ & $k_{1} / k_{2}=2$ & $k_{1} / k_{2}=3$ & $k_{1} / k_{2}=4$ & $k_{1} / k_{2}=5$ & $\begin{array}{l}\text { Upper } \\
\text { Bound }\end{array}$ \\
\hline$\theta_{1}$ & 8.00 & 8.38 & 9.85 & 12.00 & 14.15 & 15.62 & 16.00 \\
\hline$\theta_{2}$ & 3.00 & 3.38 & 4.85 & 7.00 & 9.15 & 10.62 & 11.00 \\
\hline$\omega^{*}$ & - & 0.237 & 0.479 & 0.569 & 0.479 & 0.237 & - \\
\hline
\end{tabular}

* Quadrature weights

The value of the joint distribution can be evaluated at each point $\left(k_{1}, k_{2}\right)$ and combined with the quadrature weight for each point $\theta_{k 1, k 2}$, as shown below:

$$
\gamma_{k 1, k 2}=\omega_{k 1} \omega_{k 2} \frac{\theta_{1}^{U}-\theta_{1}^{L}}{2} \frac{\theta_{2}^{U}-\theta_{2}^{L}}{2} \times \frac{1}{2 \pi} \exp \left[-\frac{\left(\theta_{1 k 1}-\theta_{1_{\text {mean }}}\right)^{2}+\left(\theta_{2 k 2}-\theta_{2 \text { mean }}\right)^{2}}{2}\right]
$$

Using the above formula we construct Table 9 with values of the joint distribution for all discrete points.

Table 9 Probability of each discrete point $\left(k_{1} k_{2}\right)$ according to joint distribution

\begin{tabular}{ccccccc}
\hline & & \multicolumn{5}{c}{$\theta_{1}$} \\
\cline { 3 - 6 } & & $k_{1}=1$ & $k_{1}=2$ & $k_{1}=2$ & $k_{1}=4$ & $k_{1}=5$ \\
\hline$k_{2}=1$ & $2.5 \mathrm{e}-7$ & $3.6 \mathrm{e}-5$ & $4.3 \mathrm{e}-4$ & $3.6 \mathrm{e}-5$ & $2.5 \mathrm{e}-7$ \\
$\theta_{2} \quad k_{2}=2$ & $3.6 \mathrm{e}-5$ & $5.0 \mathrm{e}-3$ & $6.1 \mathrm{e}-2$ & $5.0 \mathrm{e}-3$ & $3.6 \mathrm{e}-5$ \\
& $k_{2}=3$ & $4.3 \mathrm{e}-4$ & $6.1 \mathrm{e}-2$ & $7.3 \mathrm{e}-1$ & $6.1 \mathrm{e}-2$ & $4.3 \mathrm{e}-4$ \\
& $k_{2}=5$ & $3.6 \mathrm{e}-5$ & $5 . \mathrm{e}-3$ & $6.1 \mathrm{e}-2$ & $5.0 \mathrm{e}-3$ & $3.6 \mathrm{e}-5$ \\
\hline
\end{tabular}


Table 10 shows the cost function coefficients and the bounds for the decision variables.

Table 10 Cost coefficients and decision variable bounds for example 1

\begin{tabular}{|c|c|c|c|}
\hline $\begin{array}{c}\beta_{m}^{f i x} \\
{[M M U S D]}\end{array}$ & 10 & $\begin{array}{c}\text { capacity }{ }_{1 \mathrm{UP}}^{\mathrm{UP}} \\
{\left[10^{3} \text { tons/day }\right]}\end{array}$ & 7.5 \\
\hline $\begin{array}{c}\beta_{m}^{\mathrm{var}} \\
{[M M U S D]}\end{array}$ & 1 & $\begin{array}{c}\text { capacity }_{1 \mathrm{UP}}^{\mathrm{UP}} \\
{\left[10^{3} \text { tons/day }\right]}\end{array}$ & 7.5 \\
\hline $\begin{array}{c}\beta_{j, n}^{\mathrm{tank}} \\
{[M M U S D]}\end{array}$ & 1 & $\begin{array}{c}\text { capacity }_{2}^{\text {UP }} \\
{\left[10^{3} \text { tons/day }\right]}\end{array}$ & 10.5 \\
\hline $\begin{array}{c}v_{1}^{U P} \\
{\left[10^{3} \text { tons }\right]}\end{array}$ & 1.23 & $\begin{array}{c}\text { capacity }_{3}^{\mathrm{UP}} \\
{\left[10^{3} \text { tons/day }\right]}\end{array}$ & 13.5 \\
\hline $\begin{array}{c}v_{2}^{U P} \\
{\left[10^{3} \text { tons }\right]}\end{array}$ & 1.75 & & \\
\hline $\begin{array}{c}v_{3}^{U P} \\
{\left[10^{3} \text { tons }\right]}\end{array}$ & 2.25 & & \\
\hline
\end{tabular}

Results

To validate our MILP formulation for maximizing E(SF), we fix the design to match the one in the work by Straub and Grossmann (1990). In this design, all units are installed, they have no intermediate storage, and they are not subject to extra capacity additions (i.e. all units operate at their base capacity, as indicated in Table 1). The E(SF) reported in their work is 0.8132 , while the one obtained with the approach proposed in this paper is 0.8066 . Note that we are using five fixed quadrature points that might be inside or outside the feasible region, while Straub and Grossmann (1990) collocate all five quadrature points inside the feasible region, which involves solving a large NLP for the evaluation of the $\mathrm{E}(\mathrm{SF})$. The small difference in the result can therefore be attributed to the difference in the location of the collocation points.

The results that follow involve using the formulation described in this work with the data presented in Tables 5 - 9 and Figure 10. To maximize the E(SF) and to minimize the cost we determine the set of Pareto-optimal solutions by solving the proposed problem 
repeatedly for different values of available capital investment. This is equivalent to applying the $\varepsilon$-constrained method to the bi-criterion optimization problem. In this way, the capital investment, cap, in equation (52) is limited by the available amount $\overline{c a p}$. The results of maximum $\mathrm{E}(\mathrm{SF})$ for the different values of $\overline{c a p}$ (Pareto-optimal solutions) are summarized in Table 11 and Figure 11. Table 11 also indicates the computational time required to find the optimal solution for each value of capital investment $(\overline{c a p})$. The MILP model involves 404 discrete variables, 33,522 continuous variables, and 128,010 constraints. All results were obtained using the MILP solver CPLEX version 12.1, running on GAMS 23.3. The results were obtained using a $2.8 \mathrm{GHz}$ Intel Pentium 4 processor and 2.5 GB RAM. The optimality gap in CPLEX was set to $0.1 \%$. We found that this small gap reduced the computing time in about half for the instances that took longer to solve.

Table 11 Value of optimal E(SF) and computational times for different levels of available capital investment.

\begin{tabular}{ccc|ccc}
\hline $\begin{array}{c}\text { Capital } \\
\text { Investment }\end{array}$ & $\boldsymbol{E ( S F )}$ & $\begin{array}{l}\boldsymbol{C P U} \\
\text { seconds }\end{array}$ & $\begin{array}{l}\text { Capital } \\
\text { Investment }\end{array}$ & $\boldsymbol{E ( S F )}$ & $\begin{array}{c}\boldsymbol{C P U} \\
\text { seconds }\end{array}$ \\
\hline 0 & 0 & 2 & 35 & 0.964 & 602 \\
10 & 0.062 & 9 & 40 & 0.967 & 150 \\
11 & 0.818 & 12 & 45 & 0.983 & 144 \\
15 & 0.869 & 9 & 50 & 0.987 & 6 \\
20 & 0.870 & 143 & 55 & 0.988 & 4 \\
25 & 0.870 & 143 & 60 & 0.988 & 2 \\
30 & 0.870 & 257 & & & \\
\hline
\end{tabular}

As can be seen in Figure 11, the set of Pareto-optimal solutions is nonconvex and the $\mathrm{E}(\mathrm{SF})$ values increase step-wise rather than smoothly as the available capital increases. This effect is the result of having discrete probabilities for states $s$ and weights for the collocation points $\left(k_{1}, k_{2}\right)$. The state probability is $\mathrm{prob}^{s}$ and the collocation point weight is $\gamma_{k 1, k 2}$ in equation (17). 


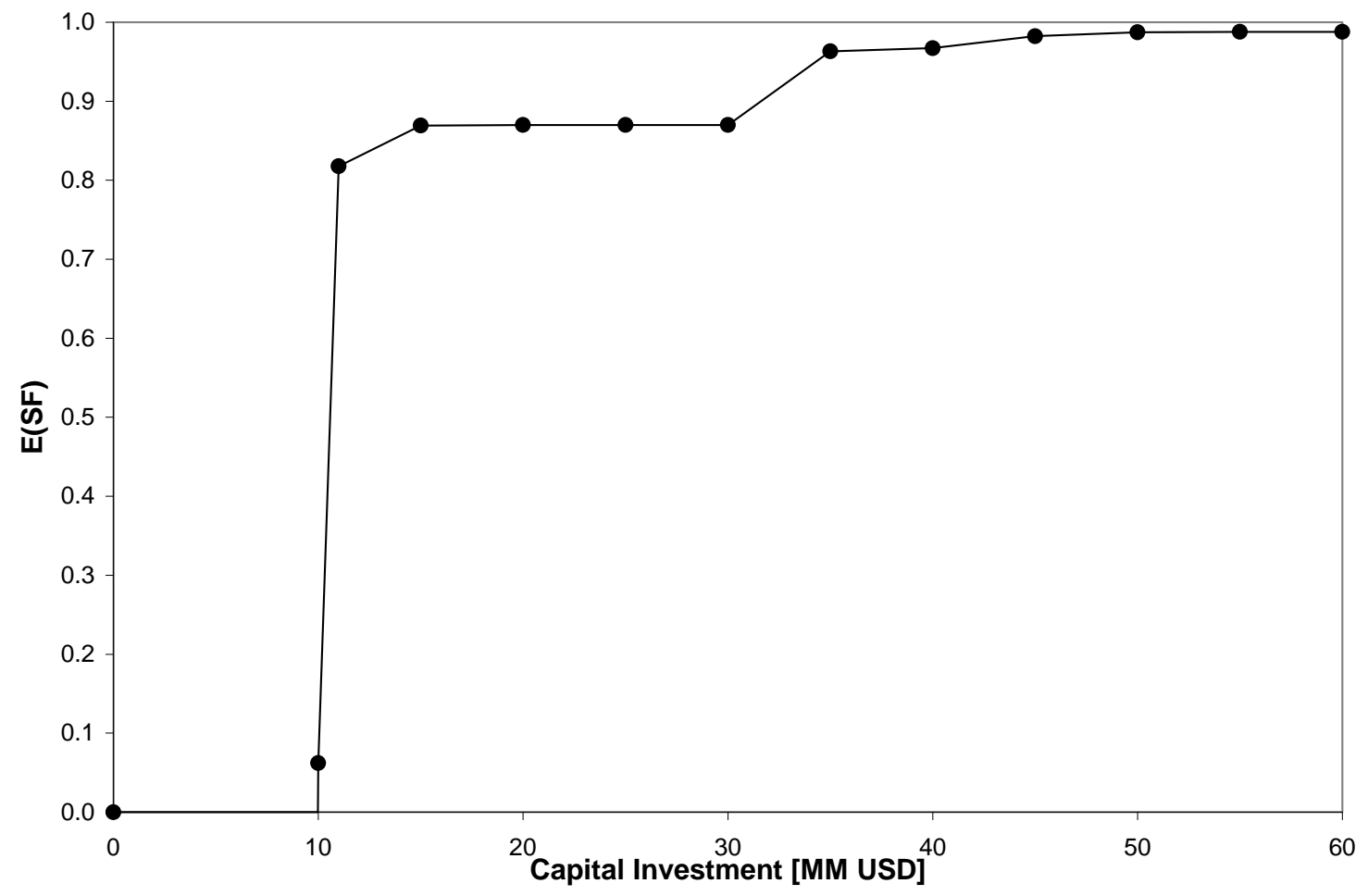

Fig. 11 Pareto curve of maximal E(SF) and minimum cost for Example 1

Table 12 presents the details of the optimal design for some values of available capital investment. Namely, the designs for 25, 35 and 45 units of available capital investment are described in this table. The capacities indicated there are calculated as base capacity (problem parameter) plus capacity addition (decision variable). This table shows the values of the linear approximation of the standard deviation of the inventory level (equation (46)), as well as the exact standard deviation calculated from equation (44). The average overestimation of standard deviation of inventory levels for the complete set of Pareto-optimal solutions presented in Table 11 is $75 \%$. The overestimation effect can be somewhat corrected by the truncation parameters $\tau_{u p}$ and $\tau_{l o}$ in equations (47) and (48). In this example we set those parameters equal to four.

In the solutions described in Table 12, storage tanks are placed after plants that directly feed the end consumer, so that the effect of any upstream failure is buffered by these storage tanks. In the low capital investment solutions, the storage tank is placed after plant 3, which is the most unreliable. When more capital becomes available a storage 
tank is also placed after plant 2 . Note also that the capacity of unit $1_{I}$ decreases when the expected stochastic flexibility increases from 0.96 to 0.98 . This is due to the addition of unit $1_{I I}$.

Table 12 Optimal design for different capital investment values. Capacity is in $10^{3}$ ton/day and the inventory volume, average level, and standard deviation in $10^{3}$ ton.

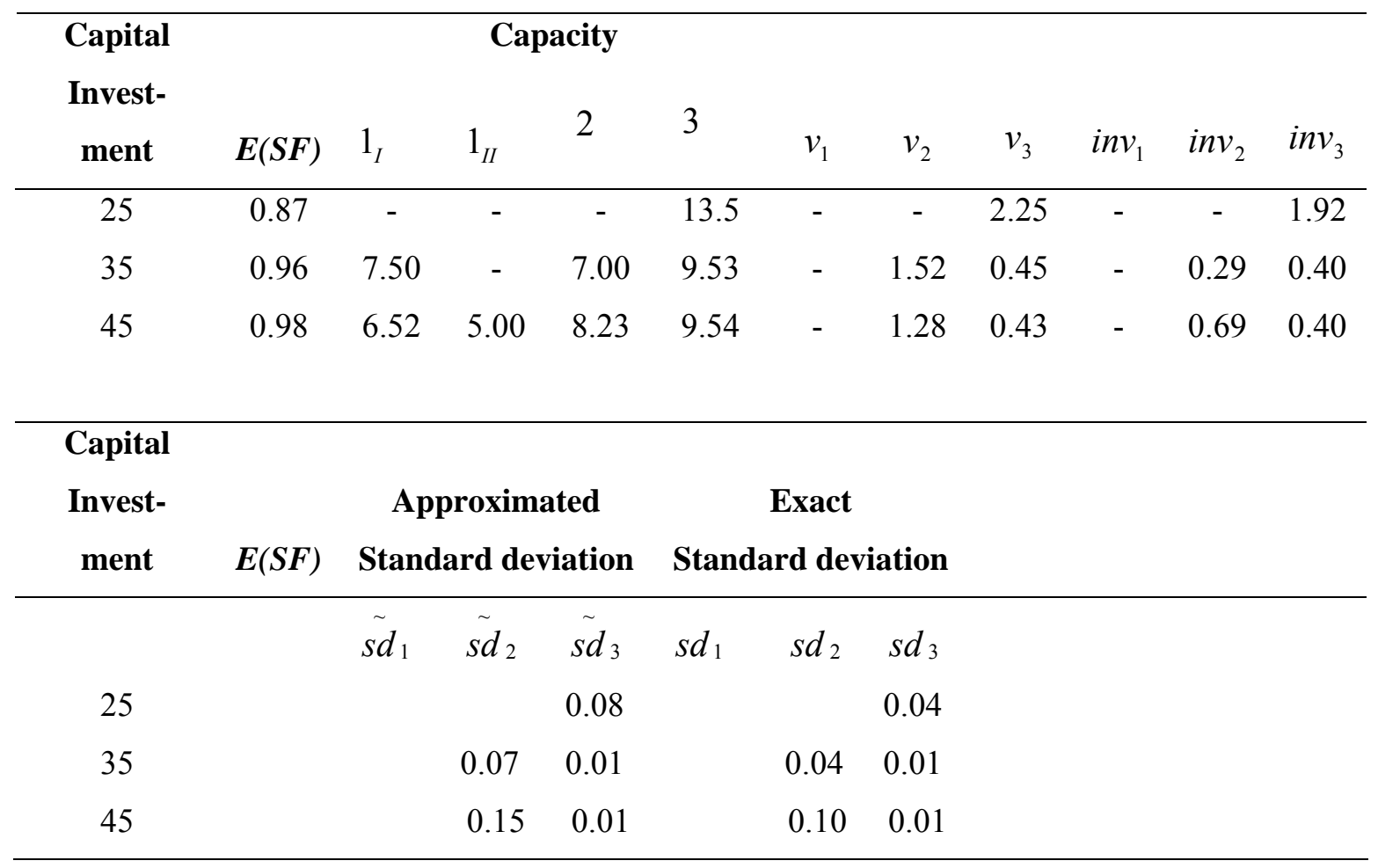

\section{Example 2}

The second example is an integrated process that consists of five interconnected chemical plants, as shown in Figure 12. The uncertainty in this example is a result of process failures with probabilistic time to failure and time to repair. There are no continuous uncertain parameters, thus, no quadrature points are required. The plants in this integrated site represent a section of a larger industrial process network. Each of these plants is modeled as a continuous process that can use one or several inputs and produce one output. The superstructure of the integrated process in Figure 12 includes two parallel production units and a storage tank for each plant, except for plant 5 where no storage tank is allowed. For confidentiality reasons, we do not provide details about 
the processes or the chemicals involved in the integrated site. Also, the capital investment values of the Pareto-optimal solutions in Figure 13 do not represent the real costs. Table 13 shows the constant rate of supply of raw materials and the constant rate of consumption of finished products. The base processing capacity and the mass balance (yield) coefficient of the units in each plant is shown in Table 14. In this example, the base processing capacities correspond to those required to satisfy the demand of products 1 and 5 when no failures occur. The mass coefficient that corresponds to $\alpha_{m}$ in equation (20) is calculated as the ratio of output over inputs. Notice that some of these coefficients are larger than one, implying that several mass units of output are produced for each mass unit of inputs. The increase in mass flow is a result of adding fillers, solvents, etc. to the product flows of each plant. We do not model directly these secondary flows but include them indirectly through the mass balance coefficients in Table 14. Likewise, waste streams not included explicitly in the process network of Figure 12 are indirectly modeled by mass balance coefficients smaller than one. The plants in the integrated site consume raw materials provided by external suppliers as well as intermediate products from other plants. Different inputs must be fed according to the stoichiometric coefficients given in Table 15. For instance, Plant 2 (second row in Table 15) requires 1.3 mass units of product 1 per unit of raw material 2.

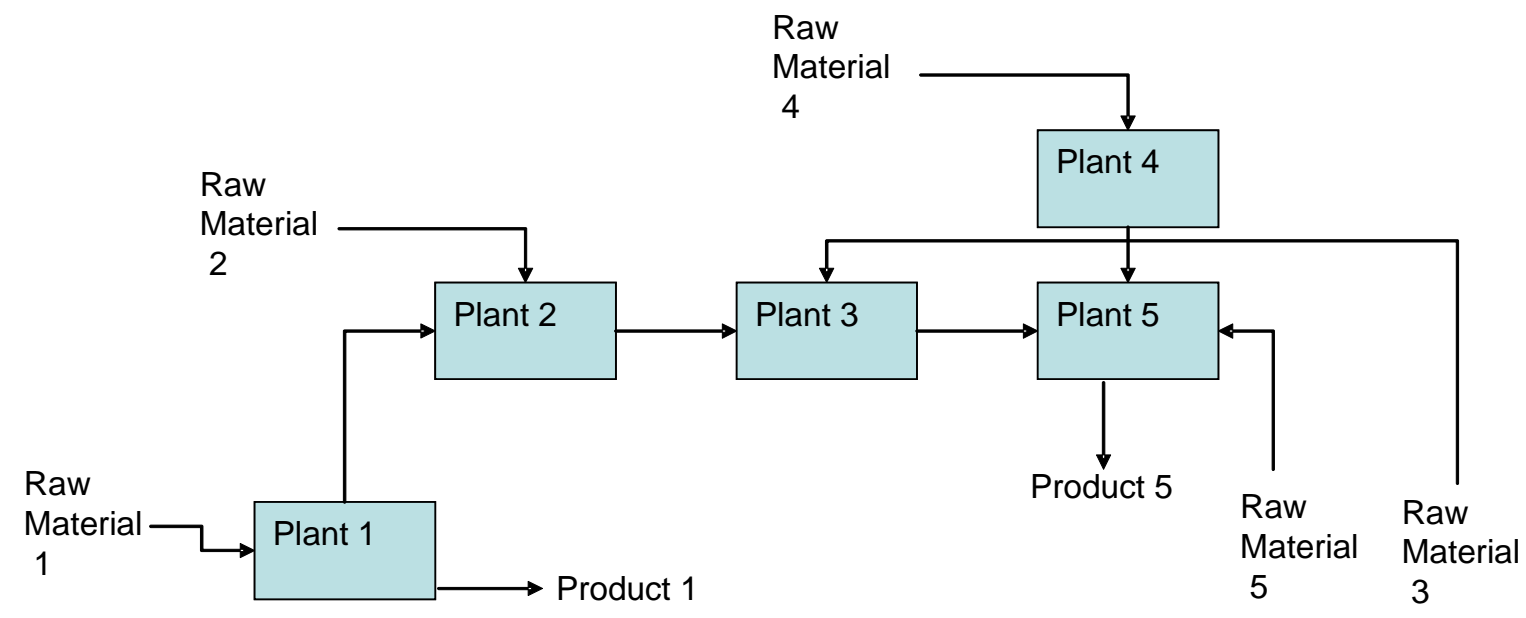

Fig. 12 Industrial integrated process 
Table 13 Supply and Demand data in industrial case study

\begin{tabular}{lccccc}
\hline $\begin{array}{l}\text { Supply of Raw Material } \\
{[\text { ton } / h]}\end{array}$ & 1 & 2 & 3 & 4 & 5 \\
\cline { 2 - 6 } & 20 & 45 & 21 & 12 & 42 \\
\hline $\begin{array}{l}\text { Demand of Product } \\
{[\text { ton } / h]}\end{array}$ & 1 & 5 & & & \\
\hline
\end{tabular}

Table 14 Base processing capacity and mass balance coefficients in industrial case study

\begin{tabular}{cccccc}
\hline & $\begin{array}{c}\text { Production } \\
\text { Units in } \\
\text { Plant } 1\end{array}$ & $\begin{array}{c}\text { Production } \\
\text { Units in } \\
\text { Plant 2 }\end{array}$ & $\begin{array}{c}\text { Production } \\
\text { Units in } \\
\text { Plant 3 }\end{array}$ & $\begin{array}{c}\text { Production } \\
\text { Units in } \\
\text { Plant 4 }\end{array}$ & $\begin{array}{c}\text { Production } \\
\text { Units in } \\
\text { Plant 5 }\end{array}$ \\
\hline $\begin{array}{c}\text { Mass } \\
\text { balance } \\
\text { coefficient }\end{array}$ & 5.29 & 0.69 & 0.70 & 3.87 & 0.46 \\
Base & & & & & \\
capacity & 13.9 & 73.4 & 53.7 & 8.3 & 109.6 \\
{$[$ ton $/ h]$} & & & & & \\
\hline
\end{tabular}

Table 15 Mass balance coefficients for the feed of external raw materials (RM) and intermediate products (P) for each plant.

\begin{tabular}{ccccccccccc}
\hline & $\mathrm{RM}_{1}$ & $\mathrm{RM}_{2}$ & $\mathrm{RM}_{3}$ & $\mathrm{RM}_{4}$ & $\mathrm{RM}_{5}$ & $\mathrm{P}_{1}$ & $\mathrm{P}_{2}$ & $\mathrm{P}_{3}$ & $\mathrm{P}_{4}$ & $\mathrm{P}_{5}$ \\
\hline Plant 1 & 1 & 0 & 0 & 0 & 0 & - & 0 & 0 & 0 & 0 \\
Plant 2 & 0 & 1 & 0 & 0 & 0 & 1.3 & - & 0 & 0 & 0 \\
Plant 3 & 0 & 0 & 1 & 0 & 0 & 0 & 17.5 & - & 0 & 0 \\
Plant 4 & 0 & 0 & 0 & 1 & 0 & 0 & 0 & 0 & - & 0 \\
Plant 5 & 0 & 0 & 0.4 & 0 & 1 & 0 & 0 & 1.3 & 1.1 & - \\
\hline
\end{tabular}


The times to failure TTF of all the units in the system are modeled using an exponential distribution, while the times to repair TTR correspond to a normal distribution. We have indicated that the Markov chain modeling technique requires exponential distributions for the TTF and TTR in order to accurately represent the transient behavior of the system. However, it is possible to obtain useful results for the stationary (long term) behavior of the system even when the TTR is represented by a normal distribution. In this case, we only need to know the mean time to repair $(M T T R)$ and use it as the mean of an exponential distribution (Billinton and Allan, 1992).

The network in Figure 12 is subjected to different types of partial and total random failures. For instance, each of the two production units in plant 2 is subject to six different types of total failures (e.g. different equipment in the unit force the production to stop), three types of partial failures that cause a $25 \%$ rate reduction, and two partial failures that cause a $10 \%$ rate reduction, resulting in 12 discrete states (11 failures plus the "no failure" state). Applying the discrete state modeling approach discussed so far in this paper results in 144 states just for the units in this plant. The total number of system states obtained from the possible combinations of discrete states of each of the units of all the plants is of the order of $5 \times 10^{10}$. To reduce this number, the failures with the same related rate reduction that occur in the same plant can be aggregated as one equivalent failure. For instance, the six different total failures in plant 2 can be modeled as one equivalent failure. Using this technique the original state space is rigorously reduced to an equivalent one with $8 \times 10^{4}$ discrete states. The fact that this is still a state space with large dimensionality emphasizes the need for future work on complexity reduction techniques based on decomposition and bounding search procedures.

In this work, to further reduce the number of states, we limit the problem to considering the 157 most probable states since these cover approximately $99 \%$ of the long term operating horizon. The MILP problem with the 157 states consists of 162 binary variables, 184,372 continuous variables, and 504,511 constraints. All results were obtained using the MILP solver CPLEX version 12.1, running on GAMS 23.3, with a 2.8 GHz Intel Pentium 4 processor and 2.5 GB RAM. A 2 percent gap between lower and upper bounds in the branch and bound algorithm was used as termination criteria for CPLEX. 
Figure 13 shows the Pareto curve of maximum E(SF) and minimum capital cost. Table 16 contains the cost coefficients used to generate this curve and the bounds on the decision variables. Table 17 shows the CPU time required to obtain each point in the curve of Figure 13, and Table 18 gives details of the optimal designs corresponding to some capital investment levels.

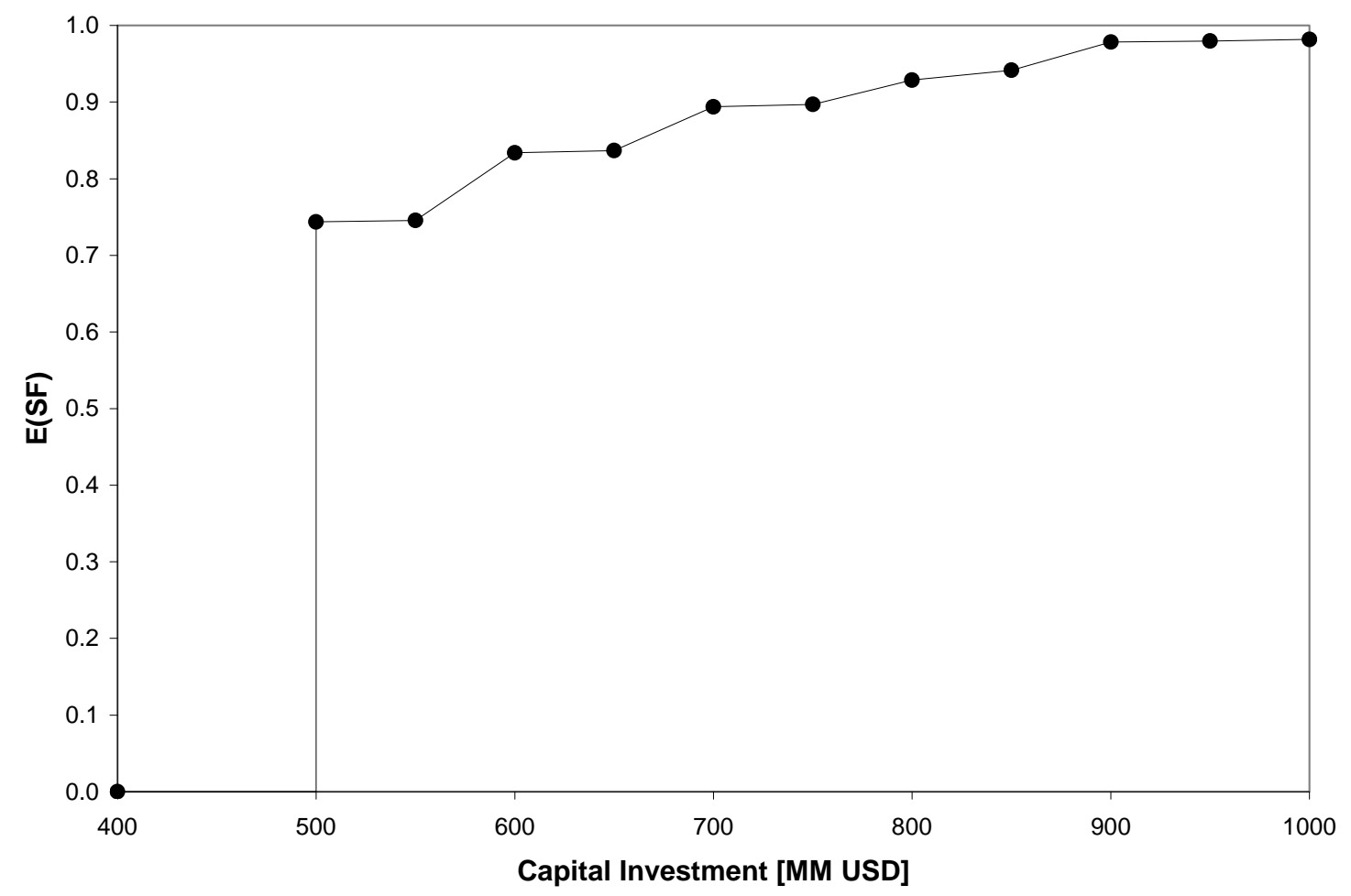

Fig. 13 Pareto curve of maximal E(SF) and minimum cost for Example 2 
Table 16 Cost coefficients and decision variable bounds for Example 2

\begin{tabular}{|c|c|c|c|}
\hline $\begin{array}{c}\beta_{m}^{f i x} \\
{[M M U S D]}\end{array}$ & 100 & $\begin{array}{c}\operatorname{capacity}_{1_{\mathrm{I}} / 1_{\mathrm{II}}}^{\mathrm{UP}} \\
{[\text { ton } / \mathrm{h}]}\end{array}$ & 20.9 \\
\hline$\beta_{m}^{\mathrm{var}}$ & 10 & $\operatorname{capacity}_{2_{\mathrm{I}} / 2_{\mathrm{II}}}^{\mathrm{UP}}$ & 110.4 \\
\hline$[M M U S D]$ & & {$[$ ton/h] } & \\
\hline$\beta_{j, n}$ & 0.005 & $\operatorname{capacity}_{3_{\mathrm{I}} / 3_{\mathrm{II}}}^{\mathrm{UP}}$ & 80.7 \\
\hline$[M M U S D]$ & & {$[$ ton $/ h]$} & \\
\hline$v_{1}^{U P}$ & 15,000 & capacity $_{4_{\mathrm{I}} / 4_{\mathrm{II}}}^{\mathrm{UP}}$ & 12.3 \\
\hline$\left[10^{3}\right.$ ton $]$ & & {$[$ ton $/ h]$} & \\
\hline$v_{2}^{U P}$ & 7,500 & capacity $_{5_{\mathrm{I}} / 5_{\mathrm{II}}}^{\mathrm{UP}}$ & 164.6 \\
\hline$\left[10^{3}\right.$ ton $]$ & & {$[$ ton $/ h]$} & \\
\hline$v_{3}^{U P}$ & 7,500 & & \\
\hline$\left[10^{3}\right.$ ton $]$ & & & \\
\hline$v_{4}^{U P}$ & 5,000 & & \\
\hline$\left[10^{3}\right.$ ton $]$ & & & \\
\hline$v_{5}^{U P}$ & 0 & & \\
\hline$\left[10^{3}\right.$ ton $]$ & & & \\
\hline
\end{tabular}

Table 17 CPU time required to obtain a solution for different capital investments.

\begin{tabular}{cc|cc}
\hline $\begin{array}{l}\text { Capital } \\
\text { Investment }\end{array}$ & $\begin{array}{l}\text { CPU } \\
\text { seconds }\end{array}$ & $\begin{array}{l}\text { Capital } \\
\text { Investment }\end{array}$ & $\begin{array}{l}\text { CPU } \\
\text { seconds }\end{array}$ \\
\hline 400 & $<1$ & 750 & 844 \\
500 & 6 & 800 & 294 \\
550 & 35 & 850 & 709 \\
600 & 202 & 900 & 338 \\
650 & 850 & 950 & 156 \\
700 & 186 & 1000 & 111 \\
\hline
\end{tabular}


Table 18 Optimal design for different capital investment values for Example 2.

\begin{tabular}{|c|c|c|c|c|c|c|c|}
\hline \multirow{2}{*}{$\begin{array}{c}\text { Capital } \\
\text { Investment }\end{array}$} & \multirow{2}{*}{$E(S F)$} & \multicolumn{6}{|c|}{ Capacity } \\
\hline & & $1_{I}$ & $1_{I I}$ & $2{ }_{I}$ & $2_{I I}$ & $3_{I}$ & $3_{I I}$ \\
\hline 500 & 0.74 & 13.9 & - & 73.4 & - & 53.7 & - \\
\hline 600 & 0.83 & 13.9 & - & 73.4 & 73.4 & 53.7 & - \\
\hline 850 & 0.94 & 13.9 & - & 73.4 & 73.4 & 53.7 & 53.7 \\
\hline \multirow[t]{3}{*}{1000} & 0.98 & 13.9 & 13.9 & 73.4 & 73.4 & 53.7 & 53.7 \\
\hline & & \multicolumn{6}{|c|}{ Capacity } \\
\hline & & $4_{I}$ & $4_{I I}$ & $5_{I}$ & $5_{I I}$ & & \\
\hline 500 & & 8.3 & - & 109.6 & - & & \\
\hline 600 & & 8.3 & - & 109.6 & - & & \\
\hline 850 & & 8.3 & - & 109.6 & 114.6 & & \\
\hline \multirow[t]{2}{*}{1000} & & 10.5 & - & 109.6 & 109.6 & & \\
\hline & & $v_{1}$ & $v_{2}$ & $v_{3}$ & $v_{4}$ & $v_{5}$ & \\
\hline 500 & & - & - & - & - & - & \\
\hline 600 & & - & - & - & - & - & \\
\hline 850 & & - & - & - & - & - & \\
\hline \multirow[t]{2}{*}{1000} & & - & 423.1 & 909.0 & 227.3 & - & \\
\hline & & $i n v_{1}$ & $i n v_{2}$ & $\operatorname{inv}_{3}$ & $\operatorname{inv}_{4}$ & $i n v_{5}$ & \\
\hline 500 & & - & - & - & - & - & \\
\hline 600 & & - & - & - & - & - & \\
\hline 850 & & - & - & - & - & - & \\
\hline 1000 & & - & 211.6 & 454.5 & 170.5 & - & \\
\hline
\end{tabular}

Notice that all plants are required as part of the integrated process, and therefore a minimum investment of 500 MM USD is required. Note that this amount represents the fixed cost of installing one unit for each plant, at the given base capacity (Table 14). The 
500 MM USD design is able to provide the required products to external consumers when all plants are running. However, when random failures are introduced the integrated site is only able to deliver the required amounts of products $74 \%$ of the time. The addition of one parallel production unit (600 MM USD design in Table 18) increases the expected stochastic flexibility to 0.83 . When multiple parallel units are installed together with intermediate storage tanks the fraction of time of feasible operation (or $\mathrm{E}(\mathrm{SF})$ ) can be increased to 0.94 (850 MM USD design in Table 18), or even to 0.98 (1000 MM USD design in Table 18).

\section{Conclusions}

In this paper, we have proposed an MILP bi-criterion formulation for optimizing the design of an integrated site subject to random failures and random supply and demand. The two optimization criteria are the maximization of the expected stochastic flexibility $\mathrm{E}(\mathrm{SF})$ and the minimization of the capital investment. The proposed formulation has several novel features that constitute the main contributions of this work. Namely, it captures the effect of random events on the levels of material in intermediate storage, and it integrates the idea of superstructure optimization in a production network with the concept of discrete states in a continuous time Markov chain. We used this formulation to solve the optimal design problem in two small networks; one adapted from the existing literature and the other provided by a major chemical company. We identified the need to address the exponential growth in problem size with the number of discrete states as a result of an increase in number of production units or failure modes per unit. In future work we will develop algorithmic techniques to deal with the growth in problem size. Also, the systematic reduction of the number of discretization points for continuous uncertain parameters, as in Novak and Kravanja (2008), represents a potential line of future work. Finally, we will incorporate realistic cost functions in order to provide meaningful solutions to industrial problems.

\section{Acknowledgement}

The authors acknowledge Naoko Akiya and Jee Park from The Dow Chemical Company for their help during the development of the formulation presented in this paper. The authors also acknowledge The Dow Chemical Company for providing financial support for this work. 


\section{References}

Bansal V., D. P. Perkins and E. N. Pistikopoulos, (2000). Flexibility analysis and design of linear systems by parametric programming. AIChE Journal, 46(2) 335 - 354.

Bansal V., D. P. Perkins and E. N. Pistikopoulos, (2002). Flexibility analysis and design using a parametric programming framework. AIChE Journal, 48(12) 2851 - 2868.

Billinton R. and R. N. Allan, Reliability Evaluation of Engineering Systems. Plenum Press, New York (1992).

Carnahan B., H. A. Luther and J. O. Wilkes, Applied Numerical Methods. Wiley, New York (1969).

Cheng L., E. Subrahmanian and A. W. Westerberg, (2003). Design and planning under uncertainty: issues on problem formulation and solution. Computers and Chemical Engineering, 27, $781-801$.

Davies K. M. and C. L. E. Swartz, (2008). MILP Formulations for Optimal Steady-State Buffer Level and Flexible Maintenance Scheduling. MASc Thesis, McMaster University.

Ehrgott M, Multicriteria Optimization. Springer Berlin, Heidelberg (2005).

Feord D., C. Jakeman and N. Shah, (2002). Modelling Multi-site Production to Optimise Customer Orders. 12 $2^{\text {th }}$ European Symposium on Computer Aided Process Engineering - ESCAPE 12, A. Povoa and H. Matos (Editors).

Goel H. D., J. Grievink and M. P. C. Weijnen, (2003). Integrated optimal reliable design, production and maintenance planning for multipurpose process plants. Computers and Chemical Engineering, 27, 1543 - 1555.

Henley E.J. and H. Hoshino, (1977). Effect of Storage Tanks on Plant Availability. Industrial and Engineering Chemistry Fundamentals, 16(4), 439 - 443.

Heyman D. P. and M. J. Sobel, Stochastic Models in Operations Research, Volume I.McGraw-Hill, New York (1982).

Hooker J. N., H. Yan, I. E. Grossmann and R. Raman, (1994). Logic cuts for processing networks with fixed charges. Computers and Operations Research, 21(3), $265-279$.

Kimm N. K., (2008). Economic and Environmental Aspects of Integration in Chemical Production Sites. PhD Thesis, Universität Karlsruhe.

Novak Pintaric Z. and Z. Kravanja (2008). Identification of critical points for the design and synthesis of flexible processes. Computers and Chemical Engineering, 32, 1603 1624. 
Orban-Mihalyko E. and B. G. Lakatos, (2004). Intermediate storage in batch/continuous processing systems under stochastic operation conditions. Computers and Chemical Engineering, 28, 2493 - 2508.

Pistikopoulos E. N., T. V. Thomaidis, A. Melin, and M.G. Ierapetritou (1996). Flexibility, reliability and maintenance considerations in batch plant design under uncertainty. Computers and Chemical Engineering, 20, S1209 - S1214.

Pistikopoulos E. N., C. G. Vassiliados, J. Arvela and L.G. Papageorgiou, (2001). Interaction of maintenance and production planning for multipurpose process plants a system effectiveness approach. Industrial and Engineering Chemistry Research, 40, $3195-3207$.

Sherali H. D., J. Desai and T. S. Glickman, (2008). Optimal Allocation of RiskReduction Resources in Event Trees. Mangement Science, 54(7), 1313 - 1321.

Straub D. A. and I. E. Grossmann, (1990). Integrated stochastic metric of flexibility for systems with discrete state and continuous parameter uncertainties. Computers and Chemical Engineering, 14(9), 967 - 985.

Straub D. A. and I. E. Grossmann, (1993). Design Optimization of Stochastic Flexibility. Computers and Chemical Engineering, 17(4), 339 - 354.

Uspensky J. V., Introduction to mathematical probability. McGraw-Hill Book Company, New York (1937).

Wassick, J. M. Enterprise-wide optimization in an integrated chemical complex. Computers and Chemical Engineering (2009), doi:10.1016/j.compchemeng.2009.06.002 


\section{Appendix}

\section{Proof of Proposition 1}

Remark Recall that $\bar{M}=\left\{m: z_{m}=1, m \in M\right\}$ refers to the set of units in the optimal flowsheet. Also, recall that the set $\bar{L}=\bigcup_{m \in \bar{M}} L_{m}$ refers to the failure modes of the units in the optimal flowsheet, and that $\bar{S}$ is the state space derived from all the combinations of simultaneous failures in $\bar{L}$.

The optimization problem can be express in a general form as:

$\max \sum_{s \in S} \operatorname{prob}^{s} w^{s}$

s.t.

$h^{s}\left(\right.$ flow $\left.^{s}, f^{s}, p s^{s}, \delta^{s}, z, d, y^{s}\right)=0 \quad \forall s \in S$

$g^{s}\left(\right.$ flow $\left.^{s}, f^{s}, p s^{s}, \delta^{s}, z, d, y^{s}\right) \leq u^{s} \forall s \in S$

$u^{s} \leq M\left(1-w^{s}\right) \quad \forall s \in S$

$w \in\{0,1\}^{|S|}$

$d \in D$

where $d$ are all the design variables other than $z$, and $D$ is a feasible set defined by the bound constraints on the design variables.

For a given parameter $y^{s}$, a design $d, z$ and a pair $p s^{s}, \delta^{s}$ we can solve $h(\cdot)$ for $f l o w^{s}, f^{s}$. Therefore, we can express the optimization problem equivalently as: $\max \sum_{s \in S} \operatorname{prob}^{s} w^{s}$

s.t.

$r^{s}\left(p s^{s}, \delta^{s}, z, d, y^{s}\right) \leq u^{s} \quad \forall s \in S$

$u^{s} \leq M\left(1-w^{s}\right) \quad \forall s \in S$

$w \in\{0,1\}^{|S|}$

$d \in D$

Let us solve the problem A.1 using the state-space $S$ derived from all the units in the superstructure and rewrite it considering only the units selected in the optimal flowsheet 
(i.e. those units $m$ such that $z_{m}=1$ ). The corresponding state-space for this flowsheet is $\bar{S}$

$\max \sum_{s \in S} \operatorname{prob}^{s} w^{s}$

s.t.

$r^{\bar{s}}\left(p s^{\bar{s}}, \delta^{\bar{s}}, d, y^{\bar{s}}\right) \leq u^{\bar{s}} \quad \forall \bar{s} \in \bar{S}$

$u^{\bar{s}} \leq M\left(1-w^{\bar{s}}\right) \quad \forall \bar{s} \in \bar{S}$

$w \in\{0,1\}^{|\bar{S}|}$

$d \in D$

We now proceed to show that the feasible regions of the variables $p s, \delta$, and $d$ are the same in problems (A.1) and (A.2).

Equations (56) and (57) enforce the condition that the feasible regions of $\delta^{s} \quad \forall s \in S_{-}$in (A.1) and $\delta^{\bar{s}} \forall \bar{s} \in \bar{S}$ in (A.2) are the same. The feasible region of $d$ is the same in (A.1) and (A.2) since the constraints that define $D$ (in $d \in D$ ) are independent of the state-space.

The feasible region for $p c^{s} \quad \forall s \in S_{-}$in (A.1) is given by non-negativity and equations (31) and (53). For the components $p c_{m}^{s} \quad \forall m \in M \backslash \bar{M}, s \in S_{-}$in (A.1) equations (53) is dominant since $z_{m}=0 \forall m \in M \backslash \bar{M}$. For the components $p c_{m}^{s} \quad \forall m \in \bar{M}, s \in S_{-}$in (A.1) equation (53) is redundant and the feasible region is given by non-negativity and equation (31).

In problem A.2 the feasible region of $p s \frac{\bar{s}}{m} \quad \forall \bar{s} \in \bar{S}, \bar{m} \in \bar{M}$ is given by non-negativity and equation (31).

Since by definition the projection of $y^{s}$ onto the state-space $\bar{S}$ is exactly $y^{\bar{s}}$, equation (31) is identical for all $m \in \bar{M}, s \in S_{-}$in (A.1) and for all $m \in \bar{M}, \bar{s} \in \bar{S}$ in (A.2). We already established that $p c_{m}^{s}=0 \forall m \in M \backslash \bar{M}, s \in S_{-}^{-}$so that the units $m \in M \backslash \bar{M}$ can be treated as non-existent in (A.1). These last two statements imply that the feasible region of $p c^{s} \forall s \in S_{-}$in (A.1) is the same as $p c^{\bar{s}} \forall \bar{s} \in S$ in (A.2). 
Given the results obtained for the feasible regions of the variables $p s, \delta$, and $d$ in (A.1) and (A.2), the functions $r^{s}(\cdot) \forall s \in S_{-}$in (A.1) and $r^{\bar{s}}(\cdot) \forall \bar{s} \in \bar{S}$ in (A.2) have the same feasible range. It then follows that the optimal solution $w^{s} \forall, s \in S_{-}$of (A.1) and $w^{\bar{s}} \quad \forall \bar{s} \in \bar{S}$ of (A.2) are identical.

Thus, $w^{s}=w^{\bar{s}} \forall, s \in S_{\bar{s}}, \bar{s} \in \bar{S}$ as defined in Proposition 1 is true.

\section{Proof of Proposition 2}

From equation (33)

$$
\operatorname{prob}^{\bar{s}}=\prod_{\substack{\ell: \bar{y}_{\ell}^{\bar{s}}=0, \ell \in \bar{L} \\ \ell: y_{\ell}^{\bar{s}}=1, \ell \in \bar{L}}} p_{\ell}\left(1-p_{\ell}\right), \bar{s} \in \bar{S}
$$

and

$$
\operatorname{prob}^{s}=\prod_{\ell: y_{\ell}^{s}=0, \ell \in L} p_{l} \prod_{\ell: y_{\ell}^{s}=1, \ell \in L}\left(1-p_{\ell}\right), \quad s \in S_{\bar{S}} .
$$

We can split the set $L$ in the multiplication of equation (A.4) into $\bar{L}$ and $L \backslash \bar{L}$ so that

$$
\operatorname{prob}^{s}=\prod_{\ell: y_{\ell}^{s}=0, \ell \in \bar{L}} p_{\ell} \prod_{\ell: y_{\ell}^{s}=1, \ell \in \bar{L}}\left(1-p_{\ell}\right) \prod_{\ell: y_{\ell}^{s}=0, \ell \in L \backslash \bar{L}} p_{\ell} \prod_{\ell: y_{\ell}^{s}=1, \ell \in L \backslash \bar{L}}\left(1-p_{\ell}\right), s \in S_{\bar{S}} .
$$

Recall that the projection of $y^{s} \in\{0,1\}^{|L|}$ onto $y^{s} \in\{0,1\}^{|\bar{L}|}=y^{\bar{s}}, \forall s \in S_{\bar{s}}, \bar{s} \in \bar{S}$. We can use this expression and substitute the multiplication over $y_{\ell}^{s}: \ell \in \bar{L}$ in (A.5) for a multiplication over $y_{\ell}^{\bar{s}}: \ell \in \bar{L}$, so:

$$
\operatorname{prob}^{s}=\prod_{\ell: y_{\ell}^{\bar{s}}=0, \ell \in \bar{L}} p_{\ell} \prod_{\ell: y_{\ell}^{\bar{s}}=1, \ell \in \bar{L}}\left(1-p_{\ell}\right) \quad \prod_{\ell: y_{\bar{l}}^{s}=0, \ell \in L \bar{L}} p_{\ell} \prod_{\ell: y_{\ell}^{s}=1, \ell \in L \bar{L}}\left(1-p_{\ell}\right), s \in S_{\bar{s}}, \bar{s} \in \bar{S},
$$

and by equation (A.3):

$$
\operatorname{prob}^{s}=\operatorname{prob}^{\bar{s}} \prod_{\ell: y_{\ell}^{s}=0, \ell \in L \bar{L} \bar{L}} p_{\ell} \prod_{\ell: y_{\ell}^{s}=1, \ell \in L \bar{L}}\left(1-p_{\ell}\right), \quad s \in S_{\bar{S}}, \bar{s} \in \bar{S}
$$

Summing over $s \in S_{\bar{S}}$,

$$
\sum_{s \in S_{\bar{s}}} \operatorname{prob}^{s}=\operatorname{prob}^{\bar{s}} \sum_{s \in S_{\bar{s}}}\left\{\prod_{\ell: y_{\ell}^{s}=0, \ell \in L \backslash \bar{L}} p_{\ell: y_{\ell}^{s}=1, \ell \in L \backslash \bar{L}}\left(1-p_{\ell}\right)\right\}, \bar{s} \in \bar{S}
$$

On the other hand, using the conditional probability notation and equation (A.7): 
$\left(\operatorname{prob}^{s} \mid \operatorname{prob}^{\bar{s}}\right)=\left(\operatorname{prob}^{\bar{s}} \prod_{\ell: y_{\ell}^{s}=0, \ell \in L \backslash \bar{L}} p_{\ell} \prod_{\ell: y_{\ell}^{s}=1, \ell \in L \backslash \bar{L}}\left(1-p_{\ell}\right) \mid \operatorname{prob}^{\bar{s}}\right), s \in S_{\bar{s}}, \bar{s} \in \bar{S}$

The conditional probability notation (A|B), which is read "A given B", in equation (A.9) implies that:

$\left(\operatorname{prob}^{s} \mid \operatorname{prob}^{\bar{s}}\right)=\prod_{\ell: y_{\ell}^{s}=0, \ell \in L \backslash \bar{L}} p_{\ell} \prod_{\ell: y_{\ell}^{s}=1, \ell \in L \backslash \bar{L}}\left(1-p_{\ell}\right), s \in S_{\bar{s}}, \bar{S} \in \bar{S}$

By its definition the collection of states $S_{-}$covers all the possible combinations of failures $l \in L \backslash \bar{L}$, and hence

$$
\sum_{s \in S_{\bar{S}}}\left(\operatorname{prob}^{s} \mid \operatorname{prob}^{\bar{s}}\right)=1 \quad \bar{s} \in \bar{S}
$$

Using equation (A.10) in (A.11),

$$
\sum_{s \in S_{\bar{S}}}\left\{\prod_{\ell: y_{\ell}^{s}=0, \ell \in L \backslash \bar{L}} p_{\ell} \prod_{\ell: y_{\ell}^{s}=1, \ell \in L \backslash \bar{L}}\left(1-p_{\ell}\right)\right\}=1
$$

Substituting equation (A.12) in equation (A.8) completes the proof.

\section{Proof of proposition 3}

$$
E(S F)^{*}=\sum_{s \in S} \operatorname{prob}^{s} w^{s} * \text { and } \overline{E(S F)^{*}}=\sum_{\bar{s} \in \bar{S}} \operatorname{prob}^{s} w^{s} *, \text { where } w^{s *} \text { is the value of } w^{s} \text { in the }
$$

optimal solution.

The set $S$ can be constructed as $S=\bigcup_{\bar{s} \in \bar{S}} S_{\bar{s}}$. It then follows that

$$
E(S F)^{*}=\sum_{\bar{s} \in \bar{S} s \in S_{s}^{-}} \sum_{\operatorname{prob}^{s} w^{s} *}
$$

By Proposition $1 \quad w^{s *}=w^{\bar{s}} * \forall s \in S_{-}, \bar{s} \in \bar{S}$.

Hence, $E(S F)^{*}=\sum_{\bar{s} \in \bar{S}} w^{\bar{s}} * \sum_{s \in S_{\bar{s}}} p_{r o b^{s}}$, and by Proposition 2,

$E(S F)^{*}=\sum_{\bar{s} \in \bar{S}} w^{\bar{s}} * \operatorname{prob}^{\bar{s}}=\overline{E(S F)} *$ and the proof is complete. 